\title{
ON THE STRUCTURE OF INTEGRAL GROUP RINGS OF SPORADIC GROUPS
}

\author{
FRAUKE M. BLEHER AND WOLFGANG KIMMERLE
}

\begin{abstract}
The object of this article is to examine a conjecture of Zassenhaus and certain variations of it for integral group rings of sporadic groups. We prove the $\mathbb{Q}$-variation and the Sylow variation for all sporadic groups and their automorphism groups. The Zassenhaus conjecture is established for eighteen of the sporadic simple groups, and for all automorphism groups of sporadic simple groups $G$ which are different from $G$. The proofs are given with the aid of the GAP computer algebra program by applying a computational procedure to the ordinary and modular character tables of the groups. It is also shown that the isomorphism problem of integral group rings has a positive answer for certain almost simple groups, in particular for the double covers of the symmetric groups.
\end{abstract}

\section{Introduction and variations on a theme of Zassenhaus}

With respect to the structure of torsion subgroups of integral group rings of finite groups the following conjecture due to Zassenhaus has been central to the research conducted over the last twenty years. The conjecture may be stated as follows. Let $G$ be a finite group.

1.1. The Zassenhaus conjecture, (ZC). Let $\mathbb{Z} G$ be the integral group ring of $G$. Denote the group of units of augmentation 1 by $V(\mathbb{Z} G)$ and let $H$ be a subgroup of $V(\mathbb{Z} G)$ of the same order as $G$; that is, $H$ is a group basis of $\mathbb{Z} G$. Then there exists a central automorphism $\sigma$ of $\mathbb{Z} G$ with $\sigma(G)=H$. In other words, $G$ and $H$ are conjugate within $\mathbb{Q} G$.

The conjecture is also of interest for more general coefficient rings than $\mathbb{Z}$. We say that (ZC) holds for a group ring $R G$, if the content of the conjecture holds in $R G$. Roggenkamp and Scott constructed a counterexample to $(\mathrm{ZC})$ of order 2880 [21, 25]. In the meantime more counterexamples have become known, and possibly the smallest counterexample has order 144 [1, 11, Satz 2.2.1]. However there is no counterexample known to the following block variations.

1.2. The block variation, $(\mathbf{B}-\mathbf{Z C})_{\mathbb{C}}$. Let $H$ be a group basis of $\mathbb{Z} G$. Let $B$ be a block of the Wedderburn decomposition of $\mathbb{C} G$. Let $\pi$ be the projection of $\mathbb{C} G$ onto $B$. Then $\pi(G)$ and $\pi(H)$ are conjugate within $B$.

1.3. The principal block variation, $(\mathbf{B}-\mathbf{Z C})_{0, p}$. Let $H$ be a group basis of $\mathbb{Z} G$. Let $p$ be a rational prime dividing $|G|$ and let $B_{1}, \ldots, B_{k}$ be the blocks of the Wedderburn decomposition of $\mathbb{C} G$ associated to the irreducible $\mathbb{C}$-characters of $G$ that belong to the principal $p$-block. Let $\pi$ be the projection of $\mathbb{C} G$ onto $B_{0}:=\oplus_{i=1}^{k} B_{i}$. Then $\pi(G)$ and $\pi(H)$ are conjugate within $B_{0}$. 
The isomorphism problem, (IP), is the question as to whether $\mathbb{Z} G \cong \mathbb{Z} H$ implies that $G \cong H$. Note that the conjecture (ZC) provides a strong answer to the isomorphism problem. However, the block variations do not in general provide a positive solution to the isomorphism problem. They do when one of the blocks involved is faithful. M. Hertweck has shown that in general the isomorphism problem has a negative solution [11]. As was pointed out by Hertweck, the counterexamples to the isomorphism problem do not supply a counterexample to $(\mathrm{B}-\mathrm{ZC})_{\mathbb{C}}$ or to $(\mathrm{B}-\mathrm{ZC})_{0, p}$. It is known that the principal block variation $(\mathrm{B}-\mathrm{ZC})_{0, p}$ is valid when $G$ is $p$-constrained, cf. $[12,24,29]$. Recall that $G$ is called $p$-constrained if $C_{G}\left(O_{p^{\prime}, p}(G) / O_{p^{\prime}}(G)\right) \leqslant O_{p^{\prime}, p}(G)$. In particular $p$-soluble groups are $p$-constrained. There are many classes of finite groups for which even (ZC) holds. For a survey see $[17,18,11]$. The results of [4], [3] and [5] indicate that it might hold for many classes of simple groups. Note that the isomorphism problem has a positive solution for simple groups [19]. Thus the Zassenhaus conjecture for such groups is a question on the automorphism group of the group ring, cf. Section 3.

We remark that the block variations stated as above also make sense modularly. It might be possible that projections of group bases of $\mathbb{Z} G$ are conjugate within the principal block of $K G$, where $K$ denotes an algebraically closed field of characteristic $p$.

Other variations of the Zassenhaus conjecture (ZC) that have been considered in recent years deal with Sylow-like theorems and class sums of $p$-elements. Let $X$ and $Y$ be group bases of $\mathbb{Z} G$.

1.4. The Sylow variation, $(\mathbf{S Y L})$. Let $S \in \operatorname{Syl}_{p}(X)$ and $T \in \operatorname{Syl}_{p}(Y)$. Then $S$ and $T$ are conjugate within $\mathbb{Q} G$.

1.5. The $p$-variation, $(\mathbf{Z C})_{p}$. There exists a group isomorphism $X \rightarrow Y$ whose $\mathbb{Z}$-linear extension preserves all class sums of $\mathbb{Z} G$ corresponding to elements of $p$-power order.

Note that if $(\mathrm{ZC})_{p}$ is satisfied, then Sylow $p$-subgroups of $X$ and $Y$ are conjugate within $\mathbb{Q} G$. Thus the difference between $(\mathrm{SYL})$ and $(\mathrm{ZC})_{p}$ is that $(\mathrm{ZC})_{p}$ implies a positive solution to (IP), while (SYL) does not. No counterexample to (SYL) is known. If $G$ is soluble then $(\mathrm{ZC})_{p}$ holds for each prime $p$ (see [20]). For other results on these variations we refer to [9] and [16].

The counterexamples to (ZC) give the impression that automorphisms coming from Galois automorphisms of the character field of a block of the Wedderburn decomposition of $\mathbb{C} G$ provide an obstruction to the validity of $(\mathrm{ZC})$. This makes it reasonable to study the following rational variation.

1.6. The $\mathbb{Q}$-variation, $(\mathbf{Z C})_{\mathbb{Q}}$. Let $X$ and $Y$ be group bases of $\mathbb{Z} G$. Then there is an automorphism $\sigma$ of $\mathbb{Q} G$ such that $\sigma(X)=Y$ and $\sigma$ fixes each component of the Wedderburn decomposition of $\mathbb{Q} G$.

Note that $(\mathrm{ZC})_{\mathbb{Q}}$ and $(\mathrm{ZC})$ coincide when $\mathbb{Q}$ is a splitting field for $G$. Clearly, $(\mathrm{ZC})_{\mathbb{Q}}$ contains a positive answer to (IP), and thus cannot hold in this form for every finite group.

If one tries to extend the above-mentioned results on the variations of the Zassenhaus conjecture from $p$-constrained groups or from soluble groups to general finite groups, then simple groups are certainly the first candidates that have to be considered. By the classification of the finite simple groups, the simple groups may be roughly divided into three types: the alternating groups, the simple groups of Lie type, and the twenty-six sporadic groups. Most of the known properties of the sporadic groups are encoded in their character tables, and it is therefore natural to deal with them via their character tables. The object of this 
paper is to give a purely character-theoretical approach to the variations of the Zassenhaus conjecture, and to apply this computer-aided method to the sporadic groups.

The paper is organized as follows. In Section 2 we state our results on the sporadic simple groups and their automorphism groups. In Section 3 we explain the reduction of the problems to the study of special automorphisms of spectral tables. In Section 4 the proofs of the results of Section 2 are given. Section 5 discusses the relationship of the defect group problem and the Green correspondence of automorphisms to our approach. Finally, in Section 6 we consider the isomorphism problem (IP) for almost simple groups. It is shown that (IP) has a positive solution for the double covers of the symmetric groups, and for all almost sporadic simple groups.

Finally, we would like to mention that we were both partially supported by the DFG Schwerpunktsprogramm 'Algorithmische Zahlentheorie und Algebra'.

\section{Results on the Zassenhaus variations}

Theorem 2.1. Let $X$ be a finite sporadic simple group with nontrivial outer automorphism group. Then $(\mathrm{ZC})$ is valid for $\operatorname{Aut}(X)=X .2$.

Theorem 2.2. The variation (SYL) is valid for all sporadic simple groups and their automorphism groups.

Theorem 2.3. The variation $(\mathrm{ZC})_{\mathbb{Q}}$ is valid for all sporadic simple groups and their automorphism groups.

Remark 2.4. Let $G$ be an alternating group. Then $(\mathrm{ZC})_{p}$ holds for $G$, cf. [18, Corollary 2.8.2]. It is also known that $(\mathrm{ZC})_{\mathbb{Q}}$ holds for $G$, cf. [15, Satz 5.9]. The proof is similar to the proof of the Zassenhaus conjecture for symmetric groups given by Peterson [23]. Therefore it remains only to establish $(\mathrm{SYL})$ and $(\mathrm{ZC})_{\mathbb{Q}}$ for simple groups of Lie type in order to prove these variations for all simple groups.

Theorem 2.5. The conjectures $(\mathrm{ZC})$ and $(\mathrm{ZC})_{p}$, for all $p$, hold for a sporadic simple group if in Table 1 the corresponding column is marked with a + sign. The third column lists all primes $p$ for which it is not known whether $(\mathrm{ZC})_{p}$ is valid. Analogously, the variations $(\mathrm{B}-\mathrm{ZC})_{\mathbb{C}}$ and $(\mathrm{B}-\mathrm{ZC})_{0, p}$, for all $p$, hold if there is $a+$ sign in the table. The last column lists all primes $p$ for which the principal block conjecture is unknown.

We note that if in the open cases the principal block conjecture $(\mathrm{B}-\mathrm{ZC})_{0, p}$ held for each prime $p$, then our results would show that the conjecture (ZC) was already valid in these open cases.

\section{The method}

In this section we describe how a computer-aided proof may be obtained to show that for a finite group the conjecture (ZC) or one of its variations is valid.

In the spirit of Brauer's famous questions the meaning of character table just means the matrix of character values and the information obtained from this matrix. So we speak of a spectral table if, in addition to the character table, the power map on the conjugacy classes 
Table 1: Results on the Zassenhaus variations

\begin{tabular}{|c|c|c|c|c|c|c|}
\hline Group & (ZC) & $\begin{array}{c}(\mathrm{ZC})_{p} \\
\text { valid } \forall p\end{array}$ & $\begin{array}{c}(\mathrm{ZC})_{p} \\
?\end{array}$ & $(\mathrm{~B}-\mathrm{ZC})_{\mathbb{C}}$ & $\begin{array}{c}(\mathrm{B}-\mathrm{ZC})_{0, p} \\
\text { valid } \forall p\end{array}$ & $\begin{array}{c}(\mathrm{B}-\mathrm{ZC})_{0, p} \\
?\end{array}$ \\
\hline $\mathrm{M}_{11}$ & + & + & - & + & + & - \\
\hline $\mathrm{M}_{12}$ & + & + & - & + & + & - \\
\hline $\mathrm{M}_{22}$ & + & + & - & + & + & - \\
\hline $\mathrm{M}_{23}$ & + & + & - & + & + & - \\
\hline $\mathrm{M}_{24}$ & + & + & - & + & + & 一 \\
\hline $\mathrm{J}_{2}$ & + & + & - & + & + & - \\
\hline Suz & + & + & - & + & + & - \\
\hline H'S & + & + & - & + & + & - \\
\hline $\mathrm{McL}$ & open & open & $p=11$ & open & open & $p=2,5$ \\
\hline $\mathrm{Co}_{3}$ & + & + & - & + & + & - \\
\hline $\mathrm{Co}_{2}$ & + & + & - & + & + & - \\
\hline $\mathrm{Co}_{1}$ & + & + & - & + & + & - \\
\hline $\mathrm{He}$ & open & + & - & + & open & $p=2,5$ \\
\hline $\mathrm{Fi}_{22}$ & open & + & - & + & open & $p=2,3$ \\
\hline $\mathrm{Fi}_{23}$ & open & open & $p=23$ & open & open & $p=2,3,5,23$ \\
\hline $\mathrm{Fi}_{24}^{\prime}$ & open & + & - & + & open & $p=2,3,23$ \\
\hline H'N & + & + & - & + & + & - \\
\hline Th & + & + & - & + & + & - \\
\hline $\mathrm{B}$ & + & + & - & + & + & - \\
\hline M & open & + & - & open & open & $p=2,3,13,17$ \\
\hline $\mathrm{J}_{1}$ & + & + & - & + & + & - \\
\hline $\mathrm{O}^{\prime} \mathrm{N}$ & + & + & - & + & + & - \\
\hline $\mathrm{J}_{3}$ & + & + & - & + & + & - \\
\hline Ly & open & open & $p=67$ & open & open & $p=3,5,67$ \\
\hline $\mathrm{Ru}$ & + & + & - & + & + & - \\
\hline $\mathrm{J}_{4}$ & open & open & $p=43$ & open & open & $p=2,11,43$ \\
\hline
\end{tabular}

(and therefore the order of representatives of the classes) is given. We denote the spectral table corresponding to the ordinary character table of a group $G$ by $\operatorname{ST}(G)$.

We should like to point out that our results rely on the correctness of the spectral tables. For our calculations we mainly used the GAP computer algebra system [27], which contains the tables of [8] and [14]. Some of the operations utilizing the spectral table may be done by hand, and for some groups - for example, the Baby Monster B - the conjecture (ZC) or one of its variations may be proved solely by looking at the spectral table. For other groups one needs substantially more information about their modular representations, and this again uses more computer algebra results, which essentially depend on computer programs.

If the isomorphism problem for $\mathbb{Z} G$ has a positive solution, then the Zassenhaus conjecture (ZC) and its variations may be studied purely in terms of automorphisms. Usually, the following conjectures in terms of automorphisms are introduced. Moreover, many results hold for a more general coefficient ring than $\mathbb{Z}$. An integral domain $S$ of characteristic zero 
is called $G$-adapted if no prime dividing $|G|$ is invertible in $S$. In particular, the Zassenhaus conjectures and their variations may also be stated for a $G$-adapted ring. A normalized ring automorphism of $S G$ is an $S$-algebra automorphism which preserves the augmentation map. The group of all normalized ring automorphisms of $S G$ is denoted by $\operatorname{Aut}_{n}(S G)$.

3.1. (AUT). Suppose that $S$ is $G$-adapted. Each $\sigma \in \operatorname{Aut}_{n}(S G)$ of $S G$ admits a Zassenhaus decomposition; that is

$$
\sigma=\alpha \cdot \tau
$$

where $\alpha$ is induced by a group automorphism of $G$ and $\tau$ is a central automorphism, which means that $\tau(c)=c$ for all $c \in Z(S G)$.

It is a consequence of the Noether-Skolem theorem that a central automorphism of $S G$ is given as conjugation with a unit of $K G$, where $K$ denotes the quotient field of $S$.

3.2. $(\mathbf{A U T})_{p}$. Suppose that $S$ is $G$-adapted. Each $\sigma \in \operatorname{Aut}_{n}(S G)$ of $S G$ can be written as

$$
\sigma=\alpha \cdot \tau,
$$

such that $\alpha$ is induced by a group automorphism of $G$, and the restriction of $\tau$ to a Sylow $p$-subgroup $P$ of $G$ is given as conjugation with a unit of $K G$.

3.3. $(\mathbf{A U T})_{\mathbb{Q}}$. Each normalized ring automorphism $\sigma$ of $\mathbb{Z} G$ can be written as

$$
\sigma=\alpha \cdot \tau,
$$

such that $\alpha$ is induced by a group automorphism of $G$, and the $\mathbb{Q}$-linear extension of $\tau$ fixes each component of the Wedderburn decomposition of $\mathbb{Q} G$.

In order to examine the Zassenhaus conjecture, one starts with an arbitrary normalized ring automorphism $\sigma$ of $S G$. Then $\sigma$ preserves the class sum correspondence; that is, $\sigma$ maps class sums to class sums. Moreover, $\sigma$ preserves the power map. Since $\sigma$ permutes the ordinary simple modules, we get the following result.

Lemma 3.1. [7, (3.4)] Let $S$ be a $G$-adapted ring. Then each $\sigma \in \operatorname{Aut}_{n}(S G)$ induces an automorphism of $\operatorname{ST}(G)$.

For convenience we again denote the induced automorphism by $\sigma$. We also use the same convention for all other induced actions of $\sigma$.

$A_{S}$ denotes the subgroup of $\operatorname{Aut}(\operatorname{ST}(G))$ induced by $\operatorname{Aut}_{n}(S G)$, and $A_{G}$ that induced by $\operatorname{Aut}(G)$. Further, we introduce the following notations. A spectral table automorphism may be written as a pair $\left(\pi_{1}, \pi_{2}\right)$, where $\pi_{1}$ and $\pi_{2}$ are permutations of $\operatorname{Irr}(G)$ and $\operatorname{Cl}(G)$ respectively, such that

$$
\pi_{1}(\chi)\left(\pi_{2}(C)\right)=\chi(C), \quad \forall \chi \in \operatorname{Irr}(G), \forall C \in \mathrm{Cl}(G) .
$$

Thus $\operatorname{Aut}(S T(G))$ induces on $\operatorname{Irr}(G)$ a permutation group which is denoted by Ach, and on $\mathrm{Cl}(G)$ a permutation group denoted by Acl. By [13, Satz V.13.5] Ach and Acl are both isomorphic to $\operatorname{Aut}(S T(G))$. The subgroups induced by $\operatorname{Aut}(G)$ are denoted by $\operatorname{Ach}_{G}$ and $\mathrm{Acl}_{G}$; those induced by $\operatorname{Aut}_{n}(S G)$, where $S$ is assumed to be $G$-adapted, are denoted by $\operatorname{Ach}_{S}$ and $\mathrm{Acl}_{S}$.

Since central automorphisms induce the identity on the spectral table, the following criteria follow now from Lemma 3.1. 
Lemma 3.2. Let $S$ be a $G$-adapted ring.

(i) $[7,3.5]$ (AUT) holds for $S G$ if, and only if,

$$
A_{S}=A_{G} .
$$

(ii) (AUT) $)_{p}$ holds for $S G$ if, and only if, for each $\sigma \in A_{S}$ there exists an $\alpha \in A_{G}$ such that $\alpha \cdot \sigma$ fixes the conjugacy class of each p-element of $G$.

(iii) $[15,5.8 \mathrm{a}]$ If $C$ is a conjugacy class of $G$ with representative $x$ and $m \in \mathbb{N}$, then $C^{m}$ denotes the conjugacy class of $x^{m}$.

$(\mathrm{AUT})_{\mathbb{Q}}$ holds for $\mathbb{Z} G$ if, and only if, for each $\sigma \in \mathrm{Acl}_{\mathbb{Z}}$ there exists an $\alpha \in \operatorname{Acl}_{G}$ such that for each conjugacy class $C$ of $G$ there exists $n_{C} \in \mathbb{N}$ with $(\alpha \cdot \sigma)(C)=C^{n_{C}}$.

The next lemma concerns the block variations.

Let $K$ be a field which is sufficiently large for $G$, and which contains $S$. If $B_{p}$ is a $p$-block then $\operatorname{Irr}\left(B_{p}\right)$ denotes the set of ordinary characters belonging to $B_{p}$. The principal $p$-block is denoted by $B_{0, p}$.

Lemma 3.3. (i) Let $B$ be a block of $K G$. Let $\chi$ be its corresponding irreducible character. Denote by $\Omega_{S}$ the orbit of $\chi$ under the action of $\mathrm{Ach}_{S}$ and by $\Omega_{G}$ the orbit under the action of $\mathrm{Ach}_{G}$. Then the projections of group bases of $S G$ isomorphic to $G$ are conjugate in $B$ if, and only if, $\Omega_{S}=\Omega_{G}$.

(ii) The following are equivalent.

(a) The block variation $(\mathrm{B}-\mathrm{ZC})_{K}$ holds for $S G$.

(b) The number of orbits of $\mathrm{Ach}_{S}$ and $\operatorname{Ach}_{G}$ on $\operatorname{Irr}(G)$ coincide.

(c) The number of orbits of $\mathrm{Acl}_{S}$ and $\mathrm{Ach}_{G}$ on $\mathrm{Cl}(G)$ coincide.

(iii) The principal block variation $(\mathrm{B}-\mathrm{ZC})_{0, p}$ holds if, and only if, $\mathrm{Ach}_{S}$ and $\mathrm{Ach}_{G}$ coincide on $\operatorname{Irr}\left(B_{0, p}\right)$.

Proof. (i) Assume that the orbits $\Omega_{S}$ and $\Omega_{G}$ coincide. Let $X$ be a group basis of $S G$ isomorphic to $G$. Let $\sigma: G \longrightarrow X$ be an isomorphism. Since $X$ and $G$ are isomorphic, there exists $\tau \in \operatorname{Aut}_{n}(S G)$ with $\tau$ restricted to $G$ coinciding with $\sigma$. Let $\psi$ be the irreducible character corresponding to $\tau(B)$. Then $\psi(\tau(g))=\chi(g)$ for each $g \in G$. The assumption on the orbits shows that there is $\alpha \in \operatorname{Aut}(G)$ with $\alpha(B)=\tau(B)$. It follows for each $g \in G$ that $\psi(\alpha(g))=\chi(g)$ and therefore that $\chi\left(\tau^{-1} \circ \alpha(g)\right)=\chi(g)$. Thus the projections of $G$ and $X$ on $B$ are conjugate within $B$.

Conversely, let $\tau \in \operatorname{Aut}_{n}(S G)$. Put $X=\tau(G)$. The projections of $X$ and $G$ are by assumption conjugate. Denote this conjugation by $\gamma$. Then $\tau^{-1} \circ \gamma$ is an automorphism of $G$. Extend this automorphism to $K G$. Then $\gamma(B)=\tau(B)$.

Part (iii) follows from part (i), as does the fact that part (ii)(a) implies part (ii)(b). The equivalence of parts (ii)(b) and (ii)(c) follows from a theorem of Brauer [13, Satz V.13.5]. Since $\mathrm{Ach}_{G}$ is a subgroup of $\mathrm{Ach}_{S}$ the orbits of both groups must already coincide, provided that their number is the same. This shows that part (ii)(b) implies part (ii)(a).

In order to apply the previous criteria, it is of course crucial to know when a spectral table automorphism comes from an automorphism of $S G$. In Section 4 we shall collect several necessary conditions. The following is a character-theoretical interpretation of [11, Proposition 2.1.1] and [25, IX 1.6] respectively. It gives a sufficient criterion in particular in the semilocal and $G$-adapted case; that is, for example when $S=\mathbb{Z}_{\pi}$ and $\pi=\pi(G)$. Note that it may also be applied when $\pi$ is smaller than $\pi(G)$. 
Proposition 3.4. Let $G$ be a finite group. Assume that $S$ is a Dedekind domain with quotient field $K$. Assume that $S$ has only finitely many maximal ideals. Let $\sigma$ be a spectral table automorphism of $\mathrm{ST}(G)$. For each prime ideal $P$ of $S$ denote its localization by $S_{P}$. Assume that there exists for each $P$ an $S_{P}$-algebra automorphism $\alpha_{P}$ of $S_{P} \otimes_{S} S G$ which induces on $K G$ the same permutation of the blocks of $K G$ as $\sigma$. Then $\sigma$ is induced by an automorphism of $S G$.

Proof. The condition that $\alpha_{P}$ induces on $K G$ the same permutation of the blocks as $\sigma$ means that for $P, Q$ prime the automorphisms $\alpha_{P}$ and $\alpha_{Q}$ differ when extended to $K G$ only by a central automorphism. By [11, Proposition 2.1.1] we get an automorphism $\alpha$ of $S G$ which differs when extended to $S_{P} G$ only by an inner automorphism from $\alpha_{P}$. Thus $\alpha$ induces $\sigma$ on the spectral table.

\section{Proof of the Zassenhaus variations}

Throughout, $S$ denotes a $G$-adapted ring. With respect to blocks with cyclic defect, we use the following results, proved in [7].

Lemma 4.1. Let $G$ be a group with cyclic Sylow p-subgroups, and let $B_{0, p}$ be the principal p-block. Then every $\sigma \in \operatorname{Aut}_{n}(S G)$ fixes every $\chi \in \operatorname{Irr}(G)$ which belongs to $B_{0, p}$ and is not exceptional for $p$.

Note that $\chi \in \operatorname{Irr}(G)$ is called exceptional for $p$ if there is another ordinary irreducible character which restricts in the same way as $\chi$ to the $p$-regular conjugacy classes.

Lemma 4.2. Let $B_{p}$ be a cyclic p-block of $G$ which is fixed by $\sigma \in \operatorname{Aut}_{n}(S G)$. Then $\sigma$ induces a graph automorphism of the Brauer tree corresponding to $B_{p}$.

For noncyclic blocks, we use the following result, proved in [3].

Lemma 4.3. Let $G$ be a finite group and let p be a rational prime. Then every $\sigma \in \operatorname{Aut}_{n}(S G)$ induces an automorphism of the p-modular character table of $G$.

As a corollary we get the following lemma.

Lemma 4.4. Let $G$ be a finite group and let $p$ be a rational prime. Let $D=\left(d_{\chi, \varphi}\right)$ be the $p$-modular decomposition matrix of $G$. Then every $\sigma \in \operatorname{Aut}_{n}(\mathbb{Z} G)$ operates on $D$ as

$$
d_{\sigma(\chi), \sigma(\varphi)}=d_{\chi, \varphi}
$$

In some cases we look at socle series of projective indecomposable modules. Here we use the following result, which follows from the fact that every normalized ring automorphism of $S G$ induces an autoequivalence of the module category.

Lemma 4.5. Let $G$ be a finite group and let $\sigma \in \operatorname{Aut}_{n}(S G)$. If $k$ is a field such that there exists a ring homomorphism $S \rightarrow k$, then $\sigma$ induces a normalized $k$-algebra automorphism of $k G$ which we again denote by $\sigma$. Let $M$ be a $k G$-module. Then $\sigma$ operates on the socle series of $M$ as

$$
\sigma\left(\operatorname{soc}_{r}(M) / \operatorname{soc}_{r-1}(M)\right) \cong \operatorname{soc}_{r}(\sigma(M)) / \operatorname{soc}_{r+1}(\sigma(M)),
$$

where $\operatorname{soc}_{1}(M)=\operatorname{soc}(M)$ and $\operatorname{soc}_{r}(M)$ is the preimage of $\operatorname{soc}\left(M / \operatorname{soc}_{r-1}(M)\right)$ under the canonical epimorphism $M \rightarrow M / \operatorname{soc}_{r-1}(M)$. 
The groups $\mathrm{M}_{11}, \mathrm{Co}_{1}, \mathrm{~B}, \mathrm{~J}_{2}$ and $\mathrm{J}_{1}$ can be treated by using only the spectral table $\mathrm{ST}(G)$ and Lemma 4.1 (see [2]). The remaining Mathieu groups and Th have already been dealt with in [7].

To prove the remaining results, we apply the following computational procedure to each group $G$. Compute $\operatorname{Aut}(\operatorname{ST}(G))$. This may easily be done with the aid of GAP. Consider then the action of a spectral table automorphism on the irreducible characters, and determine the orbits of $\operatorname{Aut}(\operatorname{ST}(G))$ on $\operatorname{Irr}(G)$. Determine with the aid of Lemmas 4.1 and 4.2 irreducible characters which must be fixed under the action of a spectral automorphism which is induced by an automorphism of $S G$. Consider the subgroup $A$ of $\operatorname{Aut}(\operatorname{ST}(G))$ which stabilizes these characters and determine the subgroup $A_{G}$, which consists of the spectral automorphisms that come from group automorphisms. Clearly, $A_{G}$ is contained in $A$. Consider now the action of elements of $A \backslash A_{G}$ on the conjugacy classes of $G$.

To apply this procedure we will write down for each group $G$ a table as follows. In the first row we state the name of $G$, its order and the number of conjugacy classes $h$. The second row consists of a set of generators for Acl, followed by the isomorphism type of $\operatorname{Aut}(\operatorname{ST}(G))$. The third row lists the orders of the conjugacy classes given in row 2 . In the case where $|\operatorname{Out}(G)|=2$, the following row shows the generators of $\operatorname{Acl}_{G}$. The next row contains a set of generators for Ach. In the following rows certain $p$-blocks are examined. For each such prime $p$, it is stated to which kind of blocks the ordinary characters in Ach belong. We give this information for each character which is the first element of a cycle of an element of Ach. If a character is marked by an $e$, this means that the character is exceptional for $p$. In cases where the Sylow $p$-subgroups are noncyclic and the $p$-modular character table is known, generators for the character table automorphisms of the $p$-modular character table are given as permutations of numbers corresponding to the conjugacy classes, called $\operatorname{Acl}_{p}$.

4.1. The group Suz. The table for Suz is given in Table 2. By Lemma 4.2, it follows, using the cyclic 5-block $B_{3}$, that the characters 7,8 and 31,32 must be moved together. The characters 31,32 correspond to the classes 32,33 , whereas the characters 7,8 correspond to the classes 14, 15, 22, 23, 38, 39. From $\mathrm{Acl}_{2}$ and Acl we conclude that (ZC) holds.

4.2. The group H'S. (See also [4].) The table for H'S is given in Table 3. From $\mathrm{Acl}_{3}$ it follows that the classes 19, 20 and 23, 24 must be moved together. Using the notation as in [32, Appendix], it follows that the operation $(19,20)(23,24)$ on the conjugacy classes corresponds to the operation $\left(49,49^{*}\right)\left(770,770^{*}\right)$ on the 3-modular Brauer characters, whereas $(15,16)$ corresponds to $\left(154_{1}, 154_{2}\right)$. In [32], the socle series of the projective covers of the Brauer characters $154_{1}$ and $154_{2}$ have respectively been determined as:

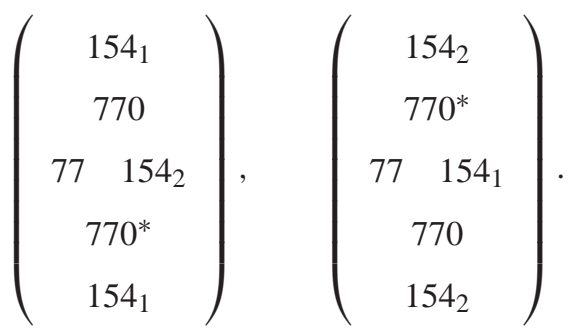

This shows that only a simultaneous operation on $\left(770,770^{*}\right)$ and $\left(154_{1}, 154_{2}\right)$ is possible. Thus the validity of (ZC) follows. 
Integral group rings of sporadic groups

Table 2: The group Suz (see paragraph 4.1)

\begin{tabular}{|c|c|c|}
\hline Suz & $2^{13} \cdot 3^{7} \cdot 5^{2} \cdot 7 \cdot 11 \cdot 13$ & $h=43$ \\
\hline Acl & $(14,15)(22,23)(38,39),(32,33),(35,36),(41,42)$ & $C_{2}^{4}$ \\
\hline orders & $6_{14}, 9_{22}, 13_{32}, 15_{35}, 18_{38}, 21_{41}$ & \\
\hline $\mathrm{Acl}_{G}$ & $(14,15)(22,23)(38,39)(32,33)(35,36)(41,42)$ & $C_{2}$ \\
\hline Ach & $(7,8)(18,19)(21,22),(31,32),(13,14),(25,26)$ & \\
\hline \multirow[t]{3}{*}{$p=5$} & $13 \mathrm{e}$ & $\in B_{2}$ defect 1 \\
\hline & 7,31 & $\in B_{3}$ defect 1 \\
\hline & $18,21,25$ & defect 0 \\
\hline $\mathrm{Acl}_{5}$ & $(14,15)(22,23)(38,39),(32,33),(41,42)$ & \\
\hline \multirow[t]{2}{*}{$p=2$} & $7,13,18 \mathrm{e}, 21,25$ & $\in B_{0}$ \\
\hline & 31 & $\in B_{1}$ defect 2 \\
\hline $\mathrm{Acl}_{2}$ & $(22,23),(32,33)(35,36)(41,42)$ & \\
\hline
\end{tabular}

Table 3: The group H'S (see paragraph 4.2)

\begin{tabular}{c|cc}
\hline H'S & $2^{9} \cdot 3^{2} \cdot 5^{3} \cdot 7 \cdot 11$ & $\mathrm{~h}=24$ \\
\hline Acl & $(15,16),(19,20),(23,24)$ & $C_{2}^{3}$ \\
orders & $8_{15}, 11_{19}, 20_{23}$ & \\
Acl $_{G}$ & $(15,16)(19,20)(23,24)$ & $C_{2}$ \\
Ach & $(5,6),(14,15),(11,12)$ & \\
$p=3$ & $5,11,14$ \\
Acl $_{3}$ & $(15,16),(19,20)(23,24)$ & \\
\hline
\end{tabular}


Integral group rings of sporadic groups

Table 4: The group McL (see paragraph 4.3)

\begin{tabular}{|c|c|c|}
\hline McL & $2^{7} \cdot 3^{6} \cdot 5^{3} \cdot 7 \cdot 11$ & $\mathrm{~h}=24$ \\
\hline Acl & $(10,11)(19,20),(13,14),(16,17),(21,22)(23,24)$ & $C_{2}^{4}$ \\
\hline orders & $7_{10}, 9_{13}, 11_{16}, 14_{19}, 15_{21}, 30_{23}$ & \\
\hline $\mathrm{Acl}_{G}$ & $(10,11)(19,20)(13,14)(21,22)(23,24)$ & $C_{2}$ \\
\hline Ach & $(16,17)(18,19),(21,22),(7,8),(5,6)(23,24)$ & \\
\hline \multirow[t]{2}{*}{$p=11$} & $7 e$ & $\in B_{0}$ \\
\hline & $5,16,18,21,23$ & defect 0 \\
\hline \multirow[t]{3}{*}{$p=7$} & $16 \mathrm{e}$ & $\in B_{0}$ \\
\hline & $18 \mathrm{e}$ & $\in B_{1}$ defect 1 \\
\hline & $5,7,21,23$ & defect 0 \\
\hline \multirow[t]{2}{*}{$p=5$} & $5 e, 7,16,21,23 \mathrm{e}$ & $\in B_{0}$ \\
\hline & 18 & defect 0 \\
\hline $\mathrm{Acl}_{5}$ & $(10,11)(19,20)(13,14),(16,17)$ & \\
\hline \multirow[t]{2}{*}{$p=3$} & $5 \mathrm{e}, 7,18,21 \mathrm{e}, 23 \mathrm{e}$ & $\in B_{0}$ \\
\hline & 16 & defect 0 \\
\hline $\mathrm{Acl}_{3}$ & $(10,11)(19,20),(16,17)$ & \\
\hline \multirow[t]{2}{*}{$p=2$} & $5,16,18,23$ & $\in B_{0}$ \\
\hline & 7,21 & defect 0 \\
\hline $\mathrm{Acl}_{2}$ & $(10,11)(21,22),(13,14),(16,17)$ & \\
\hline
\end{tabular}


4.3. The group McL. The table for McL is given in Table 4. Thus we find that either $\mathrm{Acl}_{S}=\mathrm{Acl}_{G}$ or $\mathrm{Acl}_{S}=\left\langle\mathrm{Acl}_{G},(16,17)\right\rangle$. The only possible classes that may be moved have order 11 , and these classes are linked by the power map. Consequently (AUT) $\mathbb{Q}$ is valid for $\mathrm{McL}$, and $(\mathrm{ZC})_{p}$ is valid for all $p \neq 11$. Since $A_{G}$ and $A_{S}$ have the same orbits, $(\mathrm{B}-\mathrm{ZC})_{\mathbb{C}}$ is valid. The principal block variation $(\mathrm{B}-\mathrm{ZC})_{0, p}$ holds if the characters 7 and 8 are not in $B_{0, p}$, which is the case for $p \neq 2,5$.

4.4. The group $\mathrm{Co}_{3}$. (See also [4].) The table for $\mathrm{Co}_{3}$ is given in Table 5. By Lemma 4.1, it follows, using $p=23$, that the characters 6,7 and 10,11 are fixed, and thus also the classes 24, 25, 36, 37, 38, 39. From $\mathrm{Acl}_{3}$ we conclude that (ZC) is valid.

Table 5: The group $\mathrm{Co}_{3}$ (see paragraph 4.4)

\begin{tabular}{c|cc}
\hline $\mathrm{Co}_{3}$ & $2^{10} \cdot 3^{7} \cdot 5^{3} \cdot 7 \cdot 11 \cdot 23$ & $\mathrm{~h}=42$ \\
\hline Acl & $(24,25)(36,37),(33,34),(38,39)$ & $C_{2}^{3}$ \\
orders & $11_{24}, 20_{33}, 22_{36}, 23_{38}$ & \\
Ach & $(6,7)(18,19),(10,11),(16,17)$ & \\
$p=23$ & $6,10,16 \mathrm{e}$ & $\in B_{0}$ \\
& 18 & defect 0 \\
$p=3$ & $6,10,16,18$ & $\in B_{0}$ \\
$\mathrm{Acl}_{3}$ & $(24,25)(36,37)(33,34)(38,39)$ & \\
\hline
\end{tabular}

Table 6: The group $\mathrm{Co}_{2}$ (see paragraph 4.5)

\begin{tabular}{c|cc}
\hline $\mathrm{Co}_{2}$ & $2^{18} \cdot 3^{6} \cdot 5^{3} \cdot 7 \cdot 11 \cdot 23$ & $\mathrm{~h}=60$ \\
\hline Acl & $(46,47)(59,60),(43,44),(53,54)$ & $C_{2}^{3}$ \\
orders & $1443,15_{46}, 2353,30_{59}$ & \\
Ach & $(12,13)(31,32),(22,23),(10,11)$ & \\
$p=23$ & $10 \mathrm{e}, 12,22$ & $\in B_{0}$ \\
& 31 & defect 0 \\
$p=2$ & $10,12,22 \mathrm{e}, 31$ & $\in B_{0}$ \\
Acl $_{2}$ & $(46,46)(59,60)$ & \\
\hline
\end{tabular}


4.5. The group $\mathrm{Co}_{2}$. (See also [4]. ) The table for $\mathrm{Co}_{2}$ is given in Table 6. By Lemma 4.1, it follows, using $p=23$, that the characters 12,13 and 22, 23 are fixed, and thus also the classes 46, 47, 59, 60, 43, 44. From $\mathrm{Acl}_{2}$ we conclude that (ZC) holds.

4.6. The group He. The table for He is given in Table 7. Thus we get that either $\mathrm{Acl}_{S}=$ $\mathrm{Acl}_{G}$ or

$$
\mathrm{Acl}_{S}=\langle(12,13)(15,16)(21,22)(23,24)(30,31)(32,33),(26,27)\rangle .
$$

Since all these classes are linked by the power map, $(\text { AUT })_{\mathbb{Q}}$ is valid for He. The classes 26 and 27 correspond to the characters 7 and 8 and their representatives have order 17. But an outer group automorphism interchanges these conjugacy classes. Thus it follows that $(\mathrm{ZC})_{17}$ is valid, and hence that $(\mathrm{ZC})_{p}$ holds for all $p$. Because $A_{G}$ and $A_{S}$ have the same orbits, $(\mathrm{B}-\mathrm{ZC}) \mathbb{C}$ is valid. The principal block variation is valid if the characters 7 and 8 are not in $B_{0}$. Thus it holds for $p \neq 2,5$.

4.7. The group $\mathbf{F i}_{22}$. The table for $\mathrm{Fi}_{22}$ is given in Table 8. In this case the modular considerations do not give any further restrictions for $\mathrm{Acl}_{S}$. Also, $\mathrm{Acl}$ and $\mathrm{Acl}_{5}$ coincide. $\mathrm{Acl}_{3}$ and $\mathrm{Acl}_{2}$ are not yet available in GAP. Thus $\mathrm{Acl}_{G} \leqslant \mathrm{Acl}_{S} \leqslant \mathrm{Acl}$. The orbits of Acl and $\mathrm{Acl}_{G}$ coincide. Thus $(\mathrm{B}-\mathrm{ZC})_{\mathbb{C}}$ is valid. $(\mathrm{ZC})_{p}$ is valid for all primes $p$ since for each $p$ there is at most one class involved in Acl whose representative is a $p$-element. The principal block variations are valid, except possibly for $p=2,3$.

4.8. The group $\mathbf{F i}_{23}$. The table for $\mathrm{Fi}_{23}$ is given in Table 9. The groups $\mathrm{Acl}_{2}, \mathrm{Acl}_{3}$ and $\mathrm{Acl}_{5}$ are not yet available in GAP. By Lemma 4.1 we find that

$$
1 \leqslant \mathrm{Acl}_{S} \leqslant\langle(80,81)(78,79)\rangle .
$$

Since the corresponding characters 17, 18 and 28, 29 are complex conjugate, the classes are linked by the power map. Thus $(\mathrm{AUT})_{\mathbb{Q}}$ holds. Obviously $(\mathrm{ZC})_{p}$ is valid for $p \neq 23$. $(\mathrm{B}-\mathrm{ZC})_{\mathbb{C}}$ is open for the blocks corresponding to $17,18,28$ and 29 . The principal block variation holds, except possibly for $p=2,3,5,23$.

4.9. The group $\mathbf{F i}_{24}^{\prime}$. The table for $\mathrm{Fi}_{24}^{\prime}$ is given in Table 10. The groups $\mathrm{Acl}_{2}, \mathrm{Acl}_{3}, \mathrm{Acl}_{5}$ and $\mathrm{Acl}_{7}$ are not yet available in GAP. From the table we get

$$
\left.\operatorname{Acl}_{G} \leqslant \operatorname{Acl}_{S} \leqslant\left\langle\operatorname{Acl}_{G},(106,107),(101,102),(71,72)(104) 105\right)\right\rangle .
$$

The representatives of the classes have order 45, 39, 21 and 42 . Hence $(Z C)_{p}$ is valid. Moreover, all these classes are linked by the power map. Thus (AUT) $\mathbb{Q}$ is valid for $\mathrm{Fi}_{24}^{\prime}$ It also follows that $(\mathrm{ZC})_{p}$ is valid for all $p$. Because $A_{G}$ and $A_{S}$ have the same orbits, $(\mathrm{B}-\mathrm{ZC})_{\mathbb{C}}$ is valid. The principal block variation is valid for $p=5,7,11,13,17,29 s$. Note for $p=29$ that the exceptional characters 91,92 are moved by a group automorphism. It remains open for $p=2,3,23$. For $p=23$ note that the characters 6,7 are exceptional, and are not moved by a group automorphism.

4.10. The group H'N. (See also [4].) The table for H'N is given in Table 11. By Lemma 4.1, the characters 51, 52 have to be fixed, and thus also the classes 39, 40. Using Lemma 4.2 and the cyclic 11-block $B_{1},(\mathrm{ZC})$ follows. 
Integral group rings of sporadic groups

Table 7: The group He (see paragraph 4.6)

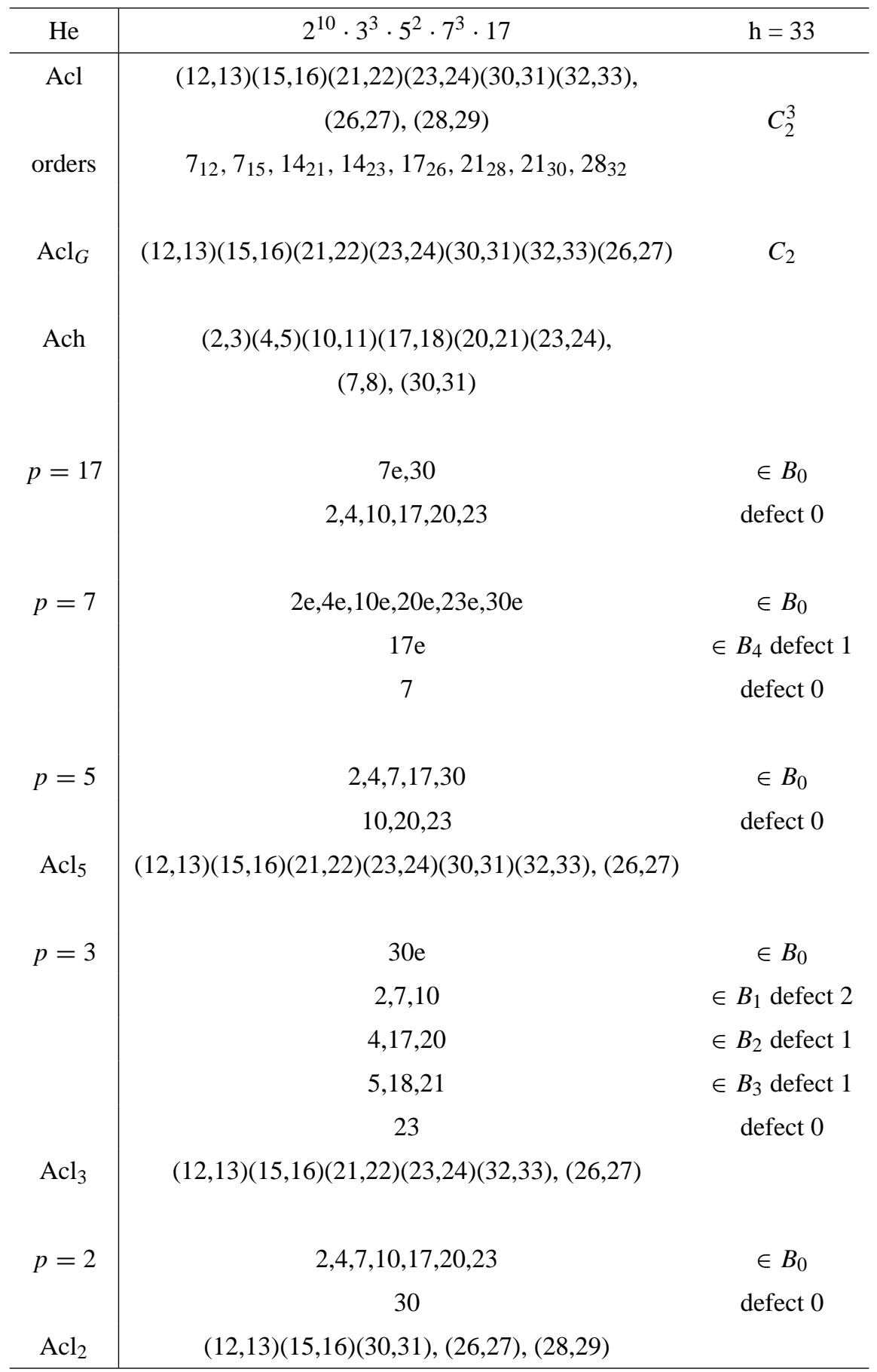


Table 8: The group $\mathrm{Fi}_{22}$ (see paragraph 4.7)

\begin{tabular}{|c|c|c|}
\hline $\mathrm{Fi}_{22}$ & $2^{17} \cdot 3^{9} \cdot 5^{2} \cdot 7 \cdot 11 \cdot 13$ & $h=65$ \\
\hline $\begin{array}{c}\text { Acl } \\
\text { orders }\end{array}$ & $\begin{array}{c}(36,37)(61,62),(42,43),(49,50),(53,54),(55,56) \\
11_{36}, 12_{42}, 13_{49}, 16_{53}, 18_{55}, 22_{61}\end{array}$ & $C_{2}^{5}$ \\
\hline $\mathrm{Acl}_{G}$ & $(36,37)(61,62)(42,43)(49,50)(53,54)(55,56)$ & $C_{2}$ \\
\hline Ach & $(40,41)(51,52),(31,32),(22,23),(33,34),(43,44)$ & \\
\hline$p=13$ & $\begin{array}{c}22 \mathrm{e} \\
31,33,40,43,51\end{array}$ & $\begin{array}{c}\in B_{0} \\
\text { defect } 0\end{array}$ \\
\hline$p=11$ & $\begin{array}{c}40 \mathrm{e} \\
51 \mathrm{e} \\
22,31,33,43\end{array}$ & $\begin{array}{c}\in B_{0} \\
\in B_{2} \text { defect } 1 \\
\text { defect } 0\end{array}$ \\
\hline$p=7$ & $22,31,33,40,43,51$ & defect 0 \\
\hline$p=5$ & $22,31,33,40,43,51$ & defect 0 \\
\hline$p=3$ & $22,31 \mathrm{e}, 33 \mathrm{e}, 40,43 \mathrm{e}, 51$ & $\in B_{0}$ \\
\hline$p=2$ & $22,31 \mathrm{e}, 33 \mathrm{e}, 40,43 \mathrm{e}, 51$ & $\in B_{0}$ \\
\hline
\end{tabular}


Integral group rings of sporadic groups

Table 9: The group $\mathrm{Fi}_{23}$ (see paragraph 4.8)

\begin{tabular}{|c|c|c|}
\hline $\mathrm{Fi}_{23}$ & $2^{18} \cdot 3^{13} \cdot 5^{2} \cdot 7 \cdot 11 \cdot 13 \cdot 17 \cdot 23$ & $\mathrm{~h}=98$ \\
\hline Acl & $(57,58)(85,86)(95,96),(63,64),(78,79),(80,81)$ & $C_{2}^{4}$ \\
\hline orders & $13_{57}, 16_{63}, 22_{78}, 23_{80}, 26_{85}, 39_{95}$ & \\
\hline Ach & $(62,63)(80,81)(94,95),(15,16),(28,29),(17,18)$ & \\
\hline \multirow[t]{2}{*}{$p=23$} & $17 \mathrm{e}, 94$ & $\in B_{0}$ \\
\hline & $15,28,62,80$ & defect 0 \\
\hline \multirow[t]{2}{*}{$p=17$} & $15,62,94$ & $\in B_{0}$ \\
\hline & $17,28,80$ & defect 0 \\
\hline \multirow[t]{4}{*}{$p=13$} & $62 \mathrm{e}$ & $\in B_{0}$ \\
\hline & $94 \mathrm{e}$ & $\in B_{2}$ defect 1 \\
\hline & $80 \mathrm{e}$ & $\in B_{5}$ defect 1 \\
\hline & $15,17,18$ & defect 0 \\
\hline \multirow[t]{3}{*}{$p=11$} & $28 \mathrm{e}$ & $\in B_{2}$ defect 1 \\
\hline & 15 & $\in B_{3}$ defect 1 \\
\hline & $17,62,80,94$ & defect 0 \\
\hline$p=7$ & $15,17,28,62,80,94$ & defect 0 \\
\hline \multirow[t]{2}{*}{$p=5$} & 28 & $\in B_{0}$ \\
\hline & $15,17,62,80,94$ & defect 0 \\
\hline$p=3$ & $15,17,28,62,80,94$ & $\in B_{0}$ \\
\hline \multirow[t]{2}{*}{$p=2$} & $15 \mathrm{e}, 17,28 \mathrm{e}, 62,80$ & $\in B_{0}$ \\
\hline & 94 & defect 0 \\
\hline
\end{tabular}


Table 10: The group $\mathrm{Fi}_{24}^{\prime}$ (see paragraph 4.9)

\begin{tabular}{|c|c|c|}
\hline $\mathrm{Fi}_{24}^{\prime}$ & $2^{21} \cdot 3^{16} \cdot 5^{2} \cdot 7^{3} \cdot 11 \cdot 13 \cdot 17 \cdot 23 \cdot 29$ & $\mathrm{~h}=108$ \\
\hline orders & $\begin{array}{c}(46,47)(78,79)(95,96),(65,66),(71,72)(104,105), \\
(74,75),(81,82),(85,86),(88,89),(92,93), \\
(99,100),(101,102),(106,107) \\
12_{46}, 18_{65}, 21_{71}, 23_{74}, 24_{78}, 24_{81}, 27_{85} \\
29_{88}, 33_{92}, 36_{95}, 39_{99}, 39_{101}, 42_{104}, 45_{106}\end{array}$ & $C_{2}^{11}$ \\
\hline $\operatorname{Acl}_{G}$ & $\begin{array}{c}(46,47)(78,79)(95,96)(65,66)(71,72)(104,105) \\
(74,75)(81,82)(85,86)(88,89)(101,102)(106,107)\end{array}$ & $C_{2}$ \\
\hline Ach & $\begin{array}{c}(39,40)(46,47)(80,81),(14,15),(60,61)(77,78), \\
(6,7),(101,102),(64,65),(91,92),(86,87), \\
(69,70),(97,98),(99,100)\end{array}$ & \\
\hline$p=29$ & $\begin{array}{c}6,86,91 \mathrm{e}, 97 \\
14,39,46,60,64,69,77,80,99,101\end{array}$ & $\begin{array}{c}\in B_{0} \\
\text { defect } 0\end{array}$ \\
\hline$p=23$ & $\begin{array}{c}6 \mathrm{e}, 97 \\
14,39,46,60,64,69,77,80,86,91,99,101\end{array}$ & $\begin{array}{c}\in B_{0} \\
\text { defect } 0\end{array}$ \\
\hline$p=17$ & $\begin{array}{c}86,97 \\
6,14,39,46,60,64,69,77,80,91,99,101\end{array}$ & $\begin{array}{c}\in B_{0} \\
\text { defect } 0\end{array}$ \\
\hline$p=13$ & $\begin{array}{c}6 \\
69 \\
14,46,39,64,91,101 \\
60,77,80,86,97,99\end{array}$ & $\begin{aligned} & \in B_{0} \\
\in & B_{4} \text { defect } 1 \\
\in & B_{8} \text { defect } 1 \\
& \text { defect } 0\end{aligned}$ \\
\hline$p=11$ & $\begin{array}{c}86 \mathrm{e} \\
6,14,39,46,60,64,69,77,80,91,97,99,101\end{array}$ & $\begin{aligned} \in & B_{3} \text { defect } 1 \\
& \text { defect } 0\end{aligned}$ \\
\hline$p=7$ & $\begin{array}{l}14,39,60,64,77,80,91 \\
6,46,69,86,97,99,101\end{array}$ & $\begin{array}{c}\in B_{0} \\
\text { defect } 0\end{array}$ \\
\hline$p=5$ & $\begin{array}{c}46,64,77,91 \\
99 \mathrm{e} \\
\text { all other }\end{array}$ & $\begin{array}{l}\in B_{2} \text { defect } 2 \\
\in B_{20} \text { defect } 1 \\
\quad \text { defect } 0\end{array}$ \\
\hline$p=3$ & $\begin{array}{c}6,14,39,46,60,64,69,77,80,86,97,99,101 \\
91\end{array}$ & $\begin{array}{c}\quad \in B_{0} \\
\in B_{2} \text { defect } 2\end{array}$ \\
\hline$p=2$ & $\begin{array}{c}6,14,39,46,60,69,77,80,86,91,99,101 \\
64 \\
97\end{array}$ & $\begin{array}{c}\in B_{0} \\
\in B_{2} \text { defect } 2 \\
\text { defect } 0\end{array}$ \\
\hline
\end{tabular}


Table 11: The group H'N (see paragraph 4.10)

\begin{tabular}{|c|c|c|}
\hline H'N & $2^{14} \cdot 3^{6} \cdot 5^{6} \cdot 7 \cdot 11 \cdot 19$ & $\mathrm{~h}=54$ \\
\hline Acl & $\begin{array}{c}(11,12)(24,25)(27,28)(35,36)(42,43)(46,47)(49,50), \\
(37,38),(39,40),(51,52),(53,54)\end{array}$ & $C_{2}^{5}$ \\
\hline orders & $\begin{array}{c}5_{11}, 10_{24}, 10_{27}, 15_{35}, 19_{37} \\
20_{39}, 20_{42}, 25_{46}, 30_{49}, 35_{51}, 40_{53}\end{array}$ & \\
\hline $\operatorname{Acl}_{G}$ & $\begin{array}{c}(11,12)(24,25)(27,28)(35,36)(42,43)(46,47)(49,50) \\
(37,38)(51,52)(53,54)\end{array}$ & $C_{2}$ \\
\hline Ach & $\begin{array}{c}(2,3)(6,7)(11,12)(13,14)(21,22)(27,28)(30,31), \\
(25,26),(51,52),(15,16),(35,36)\end{array}$ & \\
\hline$p=11$ & 51 & $\in B_{0}$ \\
\hline & $2,15,25,27,35$ & $\in B_{1}$ defect 1 \\
\hline & $6,11,13,21,30$ & defect 0 \\
\hline
\end{tabular}

4.11. The group $\mathbf{M}$. The tables for $\mathbf{M}$ are given in Tables 12 and 13 . The groups $\operatorname{Acl}_{p}$ for $p=2,3,5,7,11$ or 13 are not yet available in GAP. By Lemma 4.1 we see that $1 \leqslant \mathrm{Acl}_{S} \leqslant\langle(187,188)\rangle$. These classes have representatives of order 95 and are linked by the power map. It follows that $(\mathrm{AUT})_{\mathbb{Q}}$ and $(\mathrm{ZC})_{p}$ are valid for all $p .(\mathrm{B}-\mathrm{ZC})_{\mathbb{C}}$ holds for all blocks except the two blocks belonging to the characters 89 and 90 . Finally, the principal block variation is valid for all primes except $2,3,13,17$.

4.12. The group $\mathrm{O}^{\prime} \mathbf{N}$. The table for $\mathrm{O}^{\prime} \mathrm{N}$ is given in Table 14. By Lemma 4.1, the characters $21,22,23,24,26,27,28$ and 29, 30 are fixed, and thus also the conjugacy classes 16, 17, 22, 23, 24 and 27, 28. Further, the operation $(18,19)(20,21)$ on the classes is not possible, since the characters 21, 22 are fixed. By Lemma 4.2, it follows using the cyclic 5-block $B_{1}$ that the characters 3, 4 and 13, 14 must be moved together. The characters 3, 4 correspond to the classes 29,30, whereas the characters 13,14 correspond to the operation on the classes $(10,11)(18,20)(19,21)$. From $\mathrm{Acl}_{7}$ it follows that the classes 25, 26 and 29, 30 have to be moved together. So (ZC) follows. 
Table 12: The group M — First part (see paragraph 4.11 and Table 13)

\begin{tabular}{|c|c|c|}
\hline $\mathrm{M}$ & $\begin{array}{c}2^{46} \cdot 3^{20} \cdot 5^{9} \cdot 7^{6} \cdot 11^{2} \cdot 13^{3} \cdot 17 \cdot 19 \cdot 23 \\
\cdot 29 \cdot 31 \cdot 41 \cdot 47 \cdot 59 \cdot 71\end{array}$ & $h=194$ \\
\hline orders & $\begin{array}{c}(76,77)(135,136)(137,138)(165,166)(181,182), \\
(105,106)(160,161)(183,184),(121,122)(172,173), \\
(125,126),(132,133)(179,180),(139,140)(185,186), \\
(149,150),(152,153),(177,178),(187,188), \\
(189,190),(193,194) \\
23_{76}, 31_{105}, 39_{121}, 40_{125}, 44_{132}, 46_{135}, 46_{137}, 47_{139}, \\
56_{149}, 59_{152}, 62_{160}, 69_{165}, 71_{169}, 78_{172}, 87_{177}, \\
88_{179}, 92_{181}, 93_{183}, 94_{185}, 95_{187}, 104_{189}, 119_{193}\end{array}$ & $C_{2}^{13}$ \\
\hline Ach & $\begin{array}{c}(16,17)(55,56)(83,84)(85,86)(128,129),(102,103), \\
(26,27)(105,106)(107,108),(44,45)(99,100), \\
(41,42)(135,136),(53,54)(74,75),(81,82),(59,60) \\
\quad(39,40),(71,72),(89,90),(124,125),(47,48)\end{array}$ & \\
\hline$p=71$ & $\begin{array}{c}16,26,39 \mathrm{e}, 41,44,53,81,102,105,124,128,135 \\
47,55,59,71,74,83,85,89,99,107\end{array}$ & $\begin{array}{c}\in B_{0} \\
\text { defect } 0\end{array}$ \\
\hline$p=59$ & $\begin{array}{c}16,26,41,74,81,83,99,102 \mathrm{e}, 105,135 \\
39,44,47,53,55,59,71,85,89,107,124,128\end{array}$ & $\begin{array}{c}\in B_{0} \\
\text { defect } 0\end{array}$ \\
\hline$p=47$ & $\begin{array}{c}41,44,53 \mathrm{e}, 59,85,124 \\
16,26,71,74 \mathrm{e}, 105 \\
39,47,55,81,83,89,99,102,107,128,135\end{array}$ & $\begin{aligned} \in & B_{0} \\
\in & B_{3} \text { defect } 1 \\
& \text { defect } 0\end{aligned}$ \\
\hline$p=41$ & $\begin{array}{c}16,26,44,47,55,59,71,83,99,102,107,128 \\
39,41,53,74,81,85,89,105,124,135\end{array}$ & $\begin{array}{c}\in B_{0} \\
\text { defect } 0\end{array}$ \\
\hline$p=31$ & $\begin{array}{c}16,105 \mathrm{e} \\
55,107 \\
26,41,124 \\
39,44,47,53,59,71,74,81,83,85,89,99,102,128,135\end{array}$ & $\begin{aligned} & \in B_{0} \\
\in & B_{2} \text { defect } 1 \\
\in & B_{6} \text { defect } 1 \\
& \text { defect } 0\end{aligned}$ \\
\hline$p=29$ & $\begin{array}{c}16,39,59,85,102 \\
55,71 \\
26,41,44,47,53,74,81,83,89,99,105,107,124,128,135\end{array}$ & $\begin{aligned} \in & B_{0} \\
\in & B_{2} \text { defect } 1 \\
& \text { defect } 0\end{aligned}$ \\
\hline$p=23$ & $\begin{array}{c}41,59,83 \\
85 \\
55 \\
81,128 \\
16 \\
26,39,44,47,53,71,74,89,99,102,105,107,124,135\end{array}$ & $\begin{aligned} & \in B_{0} \\
\in & B_{2} \text { defect } 1 \\
\in & B_{3} \text { defect } 1 \\
\in & B_{4} \text { defect } 1 \\
\in & B_{7} \text { defect } 1 \\
& \text { defect } 0\end{aligned}$ \\
\hline
\end{tabular}


Table 13: The group $\mathrm{M}$ - Second part (see paragraph 4.11 and Table 12)

\begin{tabular}{|c|c|c|}
\hline $\mathrm{M}$ & $\begin{array}{c}2^{46} \cdot 3^{20} \cdot 5^{9} \cdot 7^{6} \cdot 11^{2} \cdot 13^{3} \cdot 17 \cdot 19 \cdot 23 \\
\cdot 29 \cdot 31 \cdot 41 \cdot 47 \cdot 59 \cdot 71\end{array}$ & $h=194$ \\
\hline$p=19$ & $\begin{array}{c}26,39 \\
107 \\
102,105 \\
89 \mathrm{e} \\
16,41,44,47,53,55,59,71,74,81,83,85,99,124,128,135\end{array}$ & $\begin{aligned} & \in B_{0} \\
\in & B_{2} \text { defect } 1 \\
\in & B_{3} \text { defect } 1 \\
\in & B_{4} \text { defect } 1 \\
& \text { defect } 0\end{aligned}$ \\
\hline$p=17$ & $\begin{array}{c}53,71 \\
124 \\
102 \\
47 \\
16,26,39,41,44,55,59,74,81,83,85 \\
89,99,105,107,128,135\end{array}$ & $\begin{aligned} & \in B_{0} \\
\in & B_{2} \text { defect } 1 \\
\in & B_{4} \text { defect } 1 \\
\in & B_{5} \text { defect } 1 \\
& \text { defect } 0 \\
& \text { defect } 0\end{aligned}$ \\
\hline$p=13$ & $\begin{array}{c}16,44,55,71,81,83,85,89,99 \\
128 \\
26,39,41,47,53,59,74,102,105,107,124,135\end{array}$ & $\begin{array}{l}\quad \in B_{0} \\
\in B_{2} \text { defect } 1 \\
\text { defect } 0\end{array}$ \\
\hline$p=11$ & $\begin{array}{c}44,71,89 \\
99 \\
135 \\
41 \\
16,26,39,47,53,55,59,74,81, \\
83,85,102,105,107,124,128\end{array}$ & $\begin{aligned} & \in B_{0} \\
\in & B_{2} \text { defect } 1 \\
\in & B_{6} \text { defect } 1 \\
\in & B_{9} \text { defect } 1 \\
& \text { defect } 0 \\
& \text { defect } 0\end{aligned}$ \\
\hline$p=7$ & $\begin{array}{c}26,53,59,71,74,81,102,107,124 \\
47 \\
16,39,41,44,55,83,85,89,99,105,128,135\end{array}$ & $\begin{array}{l}\in B_{0} \\
\in B_{8} \text { defect } 1 \\
\text { defect } 0\end{array}$ \\
\hline$p=5$ & $\begin{array}{c}26,39,41,44,47,59,81,99,102,105,107,124,135 \\
89 \mathrm{e} \\
16,53,55,71,74,83,85,128\end{array}$ & $\begin{array}{l}\in B_{0} \\
\in B_{7} \text { defect } 1 \\
\text { defect } 0\end{array}$ \\
\hline$p=3$ & $\begin{array}{c}16,39,41,44,47,53,55,59,74 \\
81,83,85,89,99,102,124,128,135 \\
26,105,107 \\
71\end{array}$ & $\begin{aligned} & \in B_{0} \\
& \in B_{0} \\
\in & B_{2} \text { defect } 1 \\
\in & B_{4} \text { defect } 3\end{aligned}$ \\
\hline$p=2$ & $\begin{array}{c}16,26,39,41,44,47,53,55,59,71,74,81 \\
83,85,89,99,105,107,128,135 \\
124 \\
102\end{array}$ & $\begin{aligned} & \in B_{0} \\
& \in B_{0} \\
\in & B_{4} \text { defect } 4 \\
& \text { defect } 0\end{aligned}$ \\
\hline
\end{tabular}


Table 14: The group O’N (see paragraph 4.12)

\begin{tabular}{|c|c|c|}
\hline $\mathrm{O}^{\prime} \mathrm{N}$ & $2^{9} \cdot 3^{4} \cdot 5 \cdot 7^{3} \cdot 11 \cdot 19 \cdot 31$ & $\mathrm{~h}=30$ \\
\hline Acl & $\begin{array}{c}(10,11)(18,20(19,21),(18,19)(20,21),(16,17), \\
(22,23,24),(25,26),(27,28),(29,30) \\
8_{10}, 15_{16}, 16_{18}, 19_{22}, 20_{25}, 28_{27}, 31_{29}\end{array}$ & $C_{2}^{6} \times C_{3}$ \\
\hline $\mathrm{Acl}_{G}$ & $(10,11)(18,20)(19,21)(25,26)(29,30)$ & $C_{2}$ \\
\hline Ach & $\begin{array}{c}(8,9)(13,14),(16,17)(21,22),(23,24),(26,27,28), \\
(5,6),(29,30),(3,4)\end{array}$ & \\
\hline$p=31$ & $\begin{array}{c}3 \mathrm{e}, 21,23,26,29 \\
5,8,13,16\end{array}$ & $\begin{array}{c}\in B_{0} \\
\text { defect } 0\end{array}$ \\
\hline$p=5$ & $\begin{array}{l}3,13 \\
5 \mathrm{e} \\
23 \mathrm{e}\end{array}$ & $\begin{array}{l}\in B_{1} \text { defect } 1 \\
\in B_{2} \text { defect } 1 \\
\in B_{3} \text { defect } 1\end{array}$ \\
\hline $\begin{array}{l}p=7 \\
\mathrm{Acl}_{7}\end{array}$ & $\begin{array}{c}3,5,8,13,16,21,26,29 \mathrm{e} \\
23 \\
(10,11)(18,20)(19,21),(16,17),(25,26)(29,30)\end{array}$ & $\begin{array}{c}\in B_{0} \\
\text { defect } 0\end{array}$ \\
\hline
\end{tabular}

4.13. The group $\mathbf{J}_{3}$. The table for $\mathbf{J}_{3}$ is given in Table 15 . By Lemma 4.1 , using $p=$ 19 , it follows that the characters 11,12 and 14, 15, 16 have to be fixed, and thus the classes 18, 19 and 10,11, 12 have to be fixed as well. Using the notation as in [14], it follows that the operation $(6,7)(13,14)$ on the conjugacy classes corresponds to the operation $(18 a, 18 b)(153 a, 153 b)$ on the 3 -modular Brauer characters, whereas $(20,21)$ corresponds to $(84 a, 84 b)$. In [22], the socle series of the projective covers of the Brauer characters $18 a$ and $18 b$ have been determined. We only write down the part needed for the argument:

$$
\left(\begin{array}{c}
18 a \\
\vdots \\
84 b \\
153 b \\
18 a
\end{array}\right), \quad\left(\begin{array}{c}
18 b \\
\vdots \\
84 a \\
153 a \\
18 b
\end{array}\right) .
$$

This shows that only a simultaneous action on $(18 a, 18 b)(153 a, 153 b)$ and $(84 a, 84 b)$ is possible. Thus (ZC) follows.

4.14. The group Ly. The table for Ly is given in Table 16. The groups $\operatorname{Acl}_{p}$ for $p=2,3,5$ are not yet available in GAP. Looking at the primes 67 and 37 we see by Lemma 4.1 that a normalized automorphism of SLy must fix each character except possibly 26, 27 and 28. The corresponding conjugacy classes 51,52 and 53 are linked by the power map. Hence $(\mathrm{AUT})_{\mathbb{Q}}$ holds, and $\mathrm{Acl}_{S}=1$ or $\operatorname{Acl}_{S}=\langle(51,52,53)\rangle$. Moreover $(\mathrm{ZC})_{p}$ holds for all primes $p \neq 67$. The block variation $(B-Z C) \mathbb{C}$ is valid for all characters different from 26 , 27 and 28. The principal block variation follows for the primes 2, 7, 11, 31, 37 . 
Table 15: The group $\mathrm{J}_{3}$ (see paragraph 4.13)

\begin{tabular}{c|cc}
\hline $\mathrm{J}_{3}$ & $2^{7} \cdot 3^{5} \cdot 5 \cdot 17 \cdot 19$ & $\mathrm{~h}=21$ \\
\hline Acl & $(6,7)(13,14)(16,17),(10,11,12),(18,19),(20,21)$ & $C_{2}^{3} \times C_{3}$ \\
orders & $5_{6}, 9_{10}, 10_{13}, 15_{16}, 17_{18}, 19_{20}$ & \\
Acl $_{G}$ & $(6,7)(13,14)(16,17)(20,21)$ & $C_{2}$ \\
& $(4,5)(7,8)(17,18),(14,15,16),(11,12),(2,3)$ & \\
Ach & $2 \mathrm{e}, 11,14$ & $\in B_{0}$ \\
$p=19$ & $4,7,17$ & defect 0 \\
& & \\
& $2,4,7,17,14$ & $\in B_{0}$ \\
Acl $_{3}$ & $16,7)(13,14),(18,19),(20,21)$ & defect 0 \\
\hline
\end{tabular}

4.15. The group Ru. (See also [4].) The table for Ru is given in Table 17. By Lemma 4.1, using $p=29$ and $p=7$, it follows that the characters $2,3,11,12,13,15,16,30,31$ and 34, 35 have to be fixed, and thus also the classes 25, 26, 21, 22, 23, 30, 31, 28, 29 and 35, 36. From $\mathrm{Acl}_{5}$ it follows that the remaining classes 32, 33, 34 also have to be fixed. So (ZC) follows.

4.16. The group $\mathbf{J}_{4}$. The table for $\mathbf{J}_{4}$ is shown in Table 18. The groups $\operatorname{Acl}_{p}$ with $p=$ 2, 3, 11 are not yet available in GAP. By Lemma 4.1 all irreducible characters are fixed under an automorphism coming from $S G$, except possibly the characters 17, 18 and 46, 47, 48. The permutations generating Ach, however, show that the characters 17, 18 must be moved together with the characters $2,3,4,5,6,7,9,10,12,13,15,16$. Thus $\mathrm{Ach}_{S}$ is generated by $(46,47,48)$ or $\mathrm{Ach}_{S}=1$. Since the corresponding conjugacy classes 57,58 and 59 have order 43 and are powers of each other, the variation $(Z C)_{\mathbb{Q}}$ is valid. $(Z C)_{p}$ holds for all primes except possibly $p=43$ and $(\mathrm{B}-\mathrm{ZC})_{\mathbb{C}}$ is valid for all blocks not corresponding to the characters 46, 47 and 48. The principal block variation holds for $p=3,5,7,23,29,31,37$.

Next we consider the automorphism groups of the sporadic groups which are different from the simple sporadic groups.

4.17. In the cases $\mathbf{M}_{12} .2, \mathbf{M}_{22} .2$, Suz.2, H'S.2, He.2, Fi $22.2, \mathbf{F i}_{24}^{\prime} .2, \mathbf{H}$ 'N.2, and $\mathbf{J}_{3} .2$, conjecture (ZC) follows by Lemma 4.1. In the case $\mathbf{J}_{2} .2$ the group $\mathrm{Acl}_{5}$ shows that (ZC) holds. The tables are Table 19 for $\mathrm{M}_{12} .2$, Table 20 for $\mathrm{M}_{22} .2$, Table 21 for Suz.2, Table 22 for H'S.2, Table 23 for He.2, Table 24 for $\mathrm{Fi}_{22} .2$, Table 25 for $\mathrm{Fi}_{24}^{\prime} .2$, Table 26 for H'N.2, Table 27 for $\mathrm{J}_{3} .2$, and Table 28 for $\mathrm{J}_{2} .2$. 
Table 16: The group Ly (see paragraph 4.14)

\begin{tabular}{|c|c|c|}
\hline Ly & $2^{8} \cdot 3^{7} \cdot 5^{6} \cdot 7 \cdot 11 \cdot 31 \cdot 37 \cdot 67$ & $\mathrm{~h}=53$ \\
\hline $\begin{array}{c}\text { Acl } \\
\text { orders }\end{array}$ & $\begin{array}{c}(17,18)(29,30)(43,44),(27,28)(49,50),(32,33), \\
(38,42,41,40,39),(45,46),(47,48),(51,52,53) \\
11_{17}, 21_{27}, 22_{29}, \\
24_{32}, 31_{38}, 33_{43}, 37_{45}, 40_{47}, 42_{49}, 67_{51}\end{array}$ & $C_{2}^{4} \times C_{3} \times C_{5}$ \\
\hline Ach & $\begin{array}{c}(2,3)(5,6)(7,8),(21,22)(29,30),(31,32), \\
(39,40,41,42,43),(24,25),(47,48),(26,27,28)\end{array}$ & \\
\hline$p=67$ & $\begin{array}{c}2,5,21,24,26 e, 29,31,39 \\
7,47\end{array}$ & $\begin{array}{c}\in B_{0} \\
\text { defect } 0\end{array}$ \\
\hline$p=37$ & $\begin{array}{l}2,7,24 \mathrm{e}, 39,47 \\
5,21,26,29,31\end{array}$ & $\begin{array}{c}\in B_{0} \\
\text { defect } 0\end{array}$ \\
\hline$p=31$ & $\begin{array}{c}7,39 \mathrm{e} \\
2,5,21,24,26,29,31,47\end{array}$ & $\begin{array}{c}\in B_{0} \\
\text { defect } 0\end{array}$ \\
\hline$p=11$ & $\begin{array}{c}5 \mathrm{e} \\
2 \\
7 \\
21,24,26,29,31,39,47\end{array}$ & $\begin{aligned} \in & B_{0} \\
\in & B_{2} \text { defect } 1 \\
\in & B_{4} \text { defect } 1 \\
& \text { defect } 0\end{aligned}$ \\
\hline$p=7$ & $\begin{array}{c}24 \\
47,31,2 \\
21 \\
29 \\
5,7,26,39\end{array}$ & $\begin{array}{c}\in B_{0} \\
\in B_{2} \text { defect } 1 \\
\in B_{3} \text { defect } 1 \\
\in B_{10} \text { defect } 1 \\
\text { defect } 0\end{array}$ \\
\hline$p=5$ & $\begin{array}{c}2,5,7,21,26,29,31,39,47 \\
24\end{array}$ & $\begin{array}{c}\in B_{0} \\
\text { defect } 0\end{array}$ \\
\hline$p=3$ & $\begin{array}{c}2,5,7,24,26,29,31,47 \\
21 \\
39\end{array}$ & $\begin{array}{c}\in B_{0} \\
\in B_{3} \text { defect } 1 \\
\text { defect } 0\end{array}$ \\
\hline$p=2$ & $\begin{array}{c}31,47 \\
2,5,21,24,29 \\
7,26,39\end{array}$ & $\begin{array}{c}\in B_{0} \\
\in B_{2} \text { defect } 7 \\
\text { defect } 0\end{array}$ \\
\hline
\end{tabular}


Table 17: The group Ru (see paragraph 4.15)

\begin{tabular}{c|cc}
\hline $\mathrm{Ru}$ & $2^{14} \cdot 3^{3} \cdot 5^{3} \cdot 7 \cdot 13 \cdot 29$ & $\mathrm{~h}=36$ \\
\hline Acl & $(21,22,23),(25,26),(28,29)$, & \\
& $(30,31),(32,33,34),(35,36)$ & $C_{2}^{4} \times C_{3}^{2}$ \\
orders & $14_{21}, 16_{25}, 20_{28}, 24_{30}, 2632,2935$ & \\
Ach & $(11,12,13),(2,3),(30,31)$, & \\
& $(15,16),(17,18,19),(34,35)$ & \\
$p=29$ & $2,11,15,30,34 \mathrm{e}$ & $\in B_{0}$ \\
& 17 & defect 0 \\
$p=7$ & 34 & $\in B_{0}$ \\
& $2,15,17,20$ & $\in B_{1}$ defect 1 \\
& & defect 0 \\
$p=5$ & $2,15,17,30 \mathrm{e}, 34$ & $\in B_{0}$ \\
Acl & 11 & defect 0 \\
& $(21,22,23),(25,26)$ & \\
\hline
\end{tabular}

4.18. The group McL.2. The table for McL.2 is shown in Table 29. By Lemma 4.1, using $p=7$, the characters 18,19 must be fixed. Note there are only two 7-blocks which are not of defect zero. Let $\sigma \in A_{S}$. Because the principal block is always fixed by a normalized automorphism the cyclic block $B_{1}$ is invariant under $\sigma$. Five of the vertices of the Brauer tree of $B_{1}$ belong to characters fixed by each element of $A_{S}$. Thus there is no non-trivial graph automorphism of $B_{1}$ induced by $\sigma$. By Lemma 4.2 we see that the characters 22 and 23 must be fixed by $\sigma$. Thus the classes 28, 29 and 32, 33 are fixpoints for each automorphism of $A_{S}$. From $\mathrm{Acl}_{5}$, (ZC) follows.

4.19. The group O'N.2. The table for O'N.2 is given in Table 30. By Lemma 4.1, the characters 30, 32 and 31, 33 are fixed, and thus also the classes 15, 16, 31, 32, 37, 38. From $\mathrm{Acl}_{7}$, conjecture (ZC) follows.

Proof of Theorem 2.2. The only groups for which $(\mathrm{ZC})_{p}$ is not proved by the previous arguments are $\mathrm{McL}$ with $p=11, \mathrm{Fi}_{23}$ with $p=23$, Ly with $p=67$ and $\mathrm{J}_{4}$ with $p=43$. In all these cases the Sylow $p$-subgroups are cyclic and contain their own centralizers. By [30] Sylow $p$-subgroups of group bases are conjugate within the principal $p$-block. Hence they are conjugate in $\mathbb{C} G$, and the variation (SYL) follows. 
Table 18: The group $\mathrm{J}_{4}$ (see paragraph 4.16)

\begin{tabular}{|c|c|c|}
\hline $\mathrm{J}_{4}$ & $2^{21} \cdot 3^{3} \cdot 5 \cdot 7 \cdot 11^{3} \cdot 23 \cdot 29 \cdot 31 \cdot 37 \cdot 43$ & $\mathrm{~h}=62$ \\
\hline orders & $\begin{array}{c}(12,13)(24,25)(26,27)(32,33)(39,40)(48,49)(55,56), \\
(30,31)(53,54),(37,38),(43,45,44),(46,47)(61,62), \\
(50,51,52),(57,58,59) \\
7_{12}, 14_{24}, 14_{26}, 20_{30}, 2_{32}, 24_{37}, 28_{39}, 31_{43} \\
33_{46}, 35_{48}, 37_{50}, 40_{53}, 42_{55}, 43_{57}, 66_{61}\end{array}$ & $C_{3}^{3} \times C_{2}^{4}$ \\
\hline Ach & $\begin{array}{c}(2,3)(4,5)(6,7)(9,10)(12,13)(15,16)(17,18) \\
(36,37)(38,39),(23,24),(56,57,58),(19,20)(33,34) \\
(53,54,55),(46,47,48)\end{array}$ & \\
\hline$p=43$ & $\begin{array}{c}4,9,15,46 \mathrm{e}, 53 \\
2,6,12,17,19,23,33,36,38,56\end{array}$ & $\begin{array}{c}\in B_{0} \\
\text { defect } 0\end{array}$ \\
\hline$p=37$ & $\begin{array}{c}2,15,19,23,53 \mathrm{e} \\
4,6,9,12,17,33,36,38,46,56\end{array}$ & $\begin{array}{c}\in B_{0} \\
\text { defect } 0\end{array}$ \\
\hline$p=31$ & $\begin{array}{c}36,53,56 \mathrm{e} \\
2,4,6,9,12,15,17,19,23,33,38,46\end{array}$ & $\begin{array}{c}\in B_{0} \\
\text { defect } 0\end{array}$ \\
\hline$p=29$ & $\begin{array}{c}2,6,9,12,19,33,36,38,53,56 \\
4,15,17,23,46\end{array}$ & $\begin{array}{c}\in B_{0} \\
\text { defect } 0\end{array}$ \\
\hline$p=23$ & $\begin{array}{c}2,4,6,15,23,53,56 \\
9,12,17,19,33,36,38,46\end{array}$ & $\begin{array}{c}\in B_{0} \\
\text { defect } 0\end{array}$ \\
\hline$p=11$ & $\begin{array}{c}2,4,6,9,12,17,19,23,33,36,38,46,53 \\
15,56\end{array}$ & $\begin{array}{c}\in B_{0} \\
\text { defect } 0\end{array}$ \\
\hline$p=7$ & $\begin{array}{c}6 \mathrm{e} \\
2 \\
4 \\
9 \\
12 \\
17 \\
15 \\
19,23,33,36,38,46,53,56\end{array}$ & $\begin{aligned} & \in B_{0} \\
\in & B_{2} \text { defect } 1 \\
\in & B_{3} \text { defect } 1 \\
\in & B_{4} \text { defect } 1 \\
\in & B_{5} \text { defect } 1 \\
\in & B_{6} \text { defect } 1 \\
\in & B_{7} \text { defect } 1 \\
& \text { defect } 0\end{aligned}$ \\
\hline$p=5$ & $\begin{array}{c}2,4,6,15,17 \\
12,33 \\
38 \\
36 \\
9,19,23,46,53,56\end{array}$ & $\begin{array}{l}\in B_{2} \text { defect } 1 \\
\in B_{8} \text { defect } 1 \\
\in B_{12} \text { defect } 1 \\
\in B_{17} \text { defect } 1 \\
\quad \text { defect } 0\end{array}$ \\
\hline$p=3$ & $\begin{array}{c}19 \\
2,12,17,23,383 \\
4,6,9 \\
33 \\
15,36,46,53,56\end{array}$ & $\begin{aligned} & \in B_{0} \\
\in & B_{2} \text { defect } 3 \\
\in & B_{3} \text { defect } 1 \\
\in & B_{9} \text { defect } 1 \\
& \text { defect } 0\end{aligned}$ \\
\hline$p=2$ & $\begin{array}{c}2,4,6,9,12,15,17,19,23,33,36,38,46,56 \\
53\end{array}$ & $\begin{array}{c}\in B_{0} \\
\text { defect } 0\end{array}$ \\
\hline
\end{tabular}


Table 19: The group $\mathrm{M}_{12} .2$ (see paragraph 4.17)

\begin{tabular}{c|cc}
\hline $\mathrm{M}_{12} \cdot 2$ & $2^{7} \cdot 3^{3} \cdot 5 \cdot 11$ & $\mathrm{~h}=21$ \\
\hline Acl & $(17,18),(20,21)$ & $C_{2}^{2}$ \\
orders & $10_{17}, 12_{20}$ & \\
Ach & $(7,8),(16,17)$ & \\
$p=11$ & 7,16 & $\in B_{0}$ \\
\hline
\end{tabular}

Table 20: The group $\mathrm{M}_{22} .2$ (see paragraph 4.17)

\begin{tabular}{c|cc}
\hline $\mathrm{M}_{22} \cdot 2$ & $2^{8} \cdot 3^{2} \cdot 5 \cdot 7 \cdot 11$ & $\mathrm{~h}=21$ \\
\hline Acl & $(8,9)(20,21)$ & $C_{2}$ \\
orders & $78,14_{20}$ & \\
Ach & $(5,7)(6,8)$ & \\
$p=11$ & 5,6 & $\in B_{0}$ \\
\hline
\end{tabular}

Table 21: The group Suz.2 (see paragraph 4.17)

\begin{tabular}{c|cc}
\hline Suz.2 & $2^{13} \cdot 3^{7} \cdot 5 \cdot 7 \cdot 11 \cdot 13$ & $\mathrm{~h}=68$ \\
\hline Acl & $(67,68)$ & $C_{2}$ \\
orders & $40_{67}$ & \\
Ach & $(61,62)$ & \\
$p=13$ & 61 & $\in B_{0}$ \\
\hline
\end{tabular}

Table 22: The group H'S.2 (see paragraph 4.17)

\begin{tabular}{c|cc}
\hline H'S.2 & $2^{10} \cdot 3^{2} \cdot 5^{3} \cdot 7 \cdot 11$ & $\mathrm{~h}=39$ \\
\hline Acl & $(37,38)$ & $C_{2}$ \\
orders & 2037 & \\
Ach & $(34,35)$ & \\
$p=11$ & 34 & $\in B_{0}$ \\
\hline
\end{tabular}


Table 23: The group He.2 (see paragraph 4.17)

\begin{tabular}{c|cc}
\hline He.2 & $2^{11} \cdot 3^{3} \cdot 5^{2} \cdot 7^{3} \cdot 17$ & $\mathrm{~h}=45$ \\
\hline Acl & $(23,24)(44,45),(32,33)(38,39)(41,42)$ & $C_{2}^{2}$ \\
orders & $832,16_{38}, 21_{23}, 24_{41}, 42_{44}$ & \\
Ach & $(38,40)(39,41),(22,23)(32,33)(42,43$ & \\
$p=17$ & $32,38,39,42$ & $\in B_{0}$ \\
& 22 & defect 0 \\
\hline
\end{tabular}

Table 24: The group $\mathrm{Fi}_{22} .2$ (see paragraph 4.17)

\begin{tabular}{c|cc}
\hline $\mathrm{Fi}_{22} \cdot 2$ & $2^{18} \cdot 3^{9} \cdot 5^{2} \cdot 7 \cdot 11 \cdot 13$ & $\mathrm{~h}=112$ \\
\hline Acl & $(106,107)$ & $C_{2}$ \\
orders & $24_{106}$ & \\
& & \\
Ach & $(89,90)$ & \\
degrees & $1360800_{89}$ & \\
$p=13$ & & $\in B_{0}$ \\
\hline
\end{tabular}

Table 25: The group $\mathrm{Fi}_{24}^{\prime} .2$ (see paragraph 4.17)

\begin{tabular}{c|cc}
\hline $\mathrm{Fi}_{24}^{\prime} \cdot 2$ & $2^{22} \cdot 3^{16} \cdot 5^{2} \cdot 7^{3} \cdot 11 \cdot 13 \cdot 17 \cdot 23 \cdot 29$ & $\mathrm{~h}=183$ \\
\hline Acl & $(71,72)(174,175),(85,86)(178,179)$, & \\
& $(91,92)(156,157)(181,182),(159,160)$ & $C_{2}^{4}$ \\
orders & $23_{71}, 26_{156}, 28_{159}, 33_{85}, 39_{91}, 46_{174}, 66_{178}, 78_{181}$ & \\
& & \\
Ach & $(11,13)(12,14),(147,149)(148,150)$, & \\
& $(70,71)(166,168)(167,169),(155,156)$ & \\
$p=29$ & $11,12,70,147,148,155,166,167$ & $\in B_{0}$ \\
\hline
\end{tabular}

Table 26: The group H'N.2 (see paragraph 4.17)

\begin{tabular}{c|cc}
\hline H'N.2 & $2^{15} \cdot 3^{6} \cdot 5^{4} \cdot 7 \cdot 11 \cdot 19$ & $\mathrm{~h}=78$ \\
\hline Acl & $(34,35)(73,74),(68,69),(76,77)$ & $C_{2}^{3}$ \\
orders & $20_{34}, 24_{68}, 40_{73}, 44_{76}$ & \\
& & \\
Ach & $(71,72)(73,74),(53,54),(57,58)$ & \\
$p=19$ & $53,57,73,71$ & $\in B_{0}$ \\
\hline
\end{tabular}


Integral group rings of sporadic groups

Table 27: The group $\mathrm{J}_{3} .2$ (see paragraph 4.17)

\begin{tabular}{c|cc}
\hline $\mathrm{J}_{3} .2$ & $2^{8} \cdot 3^{5} \cdot 5 \cdot 17 \cdot 19$ & $\mathrm{~h}=30$ \\
\hline $\begin{array}{c}\text { Acl } \\
\text { orders }\end{array}$ & $(9,10,11)(24,25,26),(15,16)(29,30),(27,28)$ & $C_{2}^{2} \times C_{3}$ \\
Ach & $9,17_{15}, 18_{24}, 24_{27}, 34_{29}$ & \\
& $(18,20,22)(19,21,23),(12,13)(14,15),(8,9)$ & \\
$p=19$ & $8,12,14,18,29$ & $\in B_{0}$ \\
\hline
\end{tabular}

Table 28: The group $\mathrm{J}_{2} .2$ (see paragraph 4.17)

\begin{tabular}{c|cc}
\hline $\mathrm{J}_{2} \cdot 2$ & $2^{8} \cdot 3^{3} \cdot 5^{2} \cdot 7$ & $\mathrm{~h}=27$ \\
\hline Acl & $(26,27)$ & $C_{2}$ \\
orders & $24_{26}$ & \\
& & \\
Ach & $(26,27)$ & \\
degrees & $336_{26}$ & \\
& & \\
$p=5$ & 26 & $\in B_{0}$ \\
Acl $_{5}$ & id & \\
\hline
\end{tabular}

Table 29: The group McL.2 (see paragraph 4.18)

\begin{tabular}{c|cc}
\hline McL.2 & $2^{8} \cdot 3^{6} \cdot 5^{3} \cdot 7 \cdot 11$ & $\mathrm{~h}=33$ \\
\hline Acl & $(14,15)(30,31),(28,29),(32,33)$ & $C_{2}^{3}$ \\
orders & $11_{14}, 20_{28}, 22_{30}, 24_{32}$ & \\
& & \\
Ach & $(10,12)(11,13),(18,19),(22,23)$ & \\
& 18 & $\in B_{0}$ \\
$p=7$ & 22 & $\in B_{1}$ defect 1 \\
& 10,11 & defect 0 \\
& & $\in B_{0}$ \\
Acl5 & $(14,15)(30,31)(32,33)$ & \\
\hline
\end{tabular}


Table 30: The group O’N.2 (see paragraph 4.19)

\begin{tabular}{|c|c|c|}
\hline O’N.2 & $2^{10} \cdot 3^{4} \cdot 5 \cdot 7^{3} \cdot 11 \cdot 19 \cdot 31$ & $\mathrm{~h}=45$ \\
\hline \multirow[t]{3}{*}{ Acl } & $(15,16)(31,32)(37,38)$ & \\
\hline & $(17,18)(28,29)(35,36)(42,43)(44,45)$, & \\
\hline & $(23,24)(42,44)(43,45),(19,20,21)(39,40,41)$ & $C_{2}^{3}$ \\
\hline \multirow[t]{2}{*}{ orders } & $15_{15}, 16_{17}, 19_{19}, 28_{23}, 8_{28}, 10_{31}$, & \\
\hline & $24_{35}, 30_{37}, 38_{39}, 56_{42}$ & \\
\hline \multirow[t]{3}{*}{ Ach } & $(24,25)(30,32)(31,33)$, & \\
\hline & $(7,8)(26,28)(27,29)(42,43)(44,45)$, & \\
\hline & $(12,13)(42,44)(43,45),(36,38,40)(37,39,41)$ & \\
\hline \multirow[t]{3}{*}{$p=19$} & $30,37 \mathrm{e}$ & $\in B_{0}$ \\
\hline & $31,36 \mathrm{e}$ & $\in B_{1}$ defect 1 \\
\hline & $7,12,24,26,27,42$ & defect 0 \\
\hline \multirow[t]{2}{*}{$p=7$} & $7,12 \mathrm{e}, 26,27,36,37,42 \mathrm{e}$ & $\in B_{0}$ \\
\hline & $24,30,31$ & defect 0 \\
\hline $\mathrm{Acl}_{7}$ & $(15,16)(31,32)(37,38)$ & \\
\hline
\end{tabular}

\section{Connection to the defect group problem}

Denote by $\mathbb{Z}_{p}$ the $p$-adic integers and let $B$ be a block of $\mathbb{Z}_{p} G$. Assume that $G_{B}$ and $H_{B}$ are the images of group bases $G$ and $H$ of $\mathbb{Z} G$ under the projection onto $B$. Let $D_{G}$ and $D_{H}$ be the defect groups of $G$ and $H$ respectively, with respect to the block $B$. Then the defect group problem poses the question as to whether $D_{G}$ and $D_{H}$ are conjugate in $B$, cf. [30].

With purely character-theoretical methods it does not seem to be possible to give a positive answer to this question. However the $p$-version of the Zassenhaus conjecture is closely related, as the following discussion shows.

Proposition 5.1. Assume that $(\mathrm{ZC})_{p}$ is valid for $\mathbb{Z} G$. Let $B_{0}$ be the principal block of $\mathbb{Z}_{p} G$. Let $H, D_{G}$ and $D_{H}$ be as above. Then $D_{G}$ and $D_{H}$ are conjugate within $\mathbb{Q}_{p} B_{0}:=$ $\mathbb{Q}_{p} \otimes_{\mathbb{Z}_{p}} B_{0}$.

Proof. Let $K$ be a field of characteristic zero which is sufficently large for $G$, and which contains $\mathbb{Z}_{p}$. Let $P \in \operatorname{Syl}_{p}(G)$ and let $C$ be a block of $K G$ with projection $\pi: K G \longrightarrow C$. Because $(\mathrm{ZC})_{p}$ is valid there exists a group isomorphism $\sigma: G \longrightarrow H$ such that for each $x \in P$ the characters of $(\pi \circ \sigma)(x)$ and $\pi(x)$ coincide. Thus the projections of $P$ and $\sigma(P)$ are conjugate within $C$. Let $K B_{0}:=K \otimes_{\mathbb{Z}_{p}} B_{0}=\oplus_{i=1}^{l} C_{i}$ where $C_{i}$ are blocks of $K G$. Then, conjugating simultaneously in each $C_{i}$, the projections of $P$ and $\sigma(P)$ are conjugate within $K B$. By the Noether-Deuring theorem it finally follows that the projections are already conjugate within $\mathbb{Q}_{p} B$. 
Proposition 5.2. Assume that the defect group problem has a positive answer for the principal block $B_{0}$ of $\mathbb{Z}_{p} G$. Let $N$ be the normalizer of a Sylow p-subgroup of $G$ and denote by $\operatorname{Aut}_{n}\left(B_{0}\right)$ the normalized automorphisms of $B_{0} . \operatorname{Set}_{\operatorname{Out}_{n}}\left(B_{0}\right):=\operatorname{Aut}_{n}\left(B_{0}\right) / \operatorname{Inn}\left(B_{0}\right)$. Then there is an injective group homomorphism

$$
\gamma: \operatorname{Out}_{n}\left(B_{0}\right) \longrightarrow \operatorname{Out}\left(N / O_{p^{\prime}}(N)\right) .
$$

Proof. Let $\alpha \in \operatorname{Aut}_{n}\left(B_{0}\right)$. Let $P \in \operatorname{Syl}_{p}(G)$. Because the defect groups of $\alpha(G)$ and $G$ are conjugate we can assume that $\alpha$ fixes $P$. Regard $B_{0}$ as bimodule where the action of $\mathbb{Z}_{p} G$ on the right is just multiplication in $\mathbb{Z}_{p} G$ and the action on the left is multiplication in $\mathbb{Z}_{p} G$ twisted by $\alpha$. Denote this bimodule as usual by $\alpha\left(B_{0}\right)_{1}$.

By [24, Lemma 24] the $\left(\mathbb{Z}_{p} G, \mathbb{Z}_{p} G\right)$-bimodule ${ }_{\alpha}\left(B_{0}\right)_{1}$ has a Green correspondent on $N \times$ $N$ of the form ${ }_{\beta}\left(b_{0}\right)_{1}$, where $b_{0}$ denotes the principal block of $N$ and $\beta$ is an automorphism of $b_{0}$ which restricted to $P$ coincides with $\alpha$, where we identify $P$ with its image in $b_{0}$.

$N$ is a $p$-constrained group. Thus $\mathbb{Z}_{p} N / O_{p^{\prime}}(N)=b_{0}$.

By $[29,24,12]$ the automorphism $\beta$ is the composition of a group automorphism followed by an inner automorphism. Bimodules of the form ${ }_{\delta}\left(b_{0}\right)_{1}$ where $\delta$ is an inner automorphism are isomorphic to ${ }_{1}\left(b_{0}\right)_{1}$. The usual bimodule calculus, see for example [25, VII,Lemma 1.3], shows that we may assume that $\beta$ is a group automorphism of $N / O_{p^{\prime}}(N)$. Moreover, $\beta^{-1} \circ \alpha$ restricted to $P$ is given by conjugation with a unit $v$ in $\mathbb{Z}_{p} N / O_{p^{\prime}}(N)$. By Coleman's lemma we can choose $v \in N$. Thus $\beta$ is unique up to conjugation and its restriction to $P$ coincides with the restriction of $\alpha$ to $P$ modulo an inner automorphism of $N$. Consequently the association $\alpha \mapsto \beta$ defines a map $\gamma$ from $\operatorname{Out}_{n}\left(B_{0}\right)$ to $\operatorname{Out}\left(N / O_{p^{\prime}}(N)\right)$. Suppose that $\gamma\left(\alpha_{1}\right)=\gamma\left(\alpha_{2}\right)$ then $\alpha_{2}^{-1} \circ \alpha_{1}$ restricted to $P$ is given by a conjugation of $N / O_{p^{\prime}}(N)$. Because $O_{p^{\prime}}\left(N / O_{p^{\prime}}(N)\right)=1$ and because $N / O_{p^{\prime}}(N)$ is $p$-constrained it follows from [10] that $\gamma\left(\alpha_{2}^{-1} \circ \alpha_{1}\right)=1$. The bimodule ${ }_{1}\left(b_{0}\right)_{1}$ is the Green correspondent of ${ }_{1}\left(B_{0}\right)_{1}$. Hence $\alpha_{2}^{-1} \circ \alpha_{1} \in \operatorname{Inn}\left(B_{0}\right)$.

Corollary 5.3. Assume that the finite group $G$ has a cyclic Sylow p-subgroup $P$ such that $C_{G}(x) \subset P$ for each nontrivial element $x \in P$. Let $B_{0}$ be the principal block of $\mathbb{Z}_{p} G$.

Then there is an injective group homomorphism $\gamma: \operatorname{Out}_{n}\left(B_{0}\right) \longrightarrow \operatorname{Out}(N)$, where $N$ denotes the normalizer of $P$ in $G$.

Proof. Let $\alpha \in \operatorname{Aut}_{n}\left(B_{0}\right)$. The condition on the centralizers shows that $P$ is a T.I. set. In particular $O_{p^{\prime}}(N)=1$ and the corollary follows from Proposition 5.2 because by [30, Theorem] Sylow $p$-subgroups of $G$ and $\alpha(G)$ are conjugate in $B_{0}$.

Remark 5.4. (a) Corollary 5.3 might hold much more generally. The assumption on the Sylow subgroups being cyclic may be superfluous.

(b) Assume that $G$ has trivial center. So we may regard $G$ as subgroup of $\operatorname{Aut}(G)$. If the image of $\gamma$ consists of automorphisms of $N$ given by conjugation with elements of $N_{\text {Aut }(G)}(N)$, then the principal block conjecture $(\mathrm{B}-\mathrm{ZC})_{0, p}$ holds.

However, in the situation of the sporadic simple groups Corollary 5.3 gives no additional information to the methods used in Section 4. For example, consider $G=\mathrm{Ly}$ and the prime $p=67$. In this case $N$ is the semidirect product of $P$ with a cyclic group of order 22. The assumptions of Corollary 5.3 are satisfied. Out $(N)=C_{3}$. Now the table for Ly in Section 4 shows that $\operatorname{Out}_{n}\left(B_{0}\right)$ is trivial or acts on $\operatorname{Irr}\left(B_{0,67}\right)$ as $\langle(26,27,28)\rangle$. But Out(Ly) is trivial. 


\section{The isomorphism problem for almost simple groups}

Following Aschbacher, a finite group $G$ is called almost simple if its generalized fitting subgroup $F^{*}(G)$ is quasisimple. Thus almost simple groups $G$ are precisely those groups with a normal series

$$
1<Z<K<G
$$

such that $K / Z$ is simple nonabelian, $K$ is perfect, $Z$ is central in $K$ and $G / Z$ embeds into $\operatorname{Aut}(K / Z) . G$ is called almost sporadic if $K / Z$ is a sporadic simple group. The goal of this section is to show that the isomorphism problem has a positive answer for an almost sporadic simple group, and for the double covers of the symmetric groups.

Our results indicate that the isomorphism problem might be true for all almost simple groups. Note also that in [6] it is shown that (IP) holds for finite groups of Lie type arising from simply connected algebraic groups over an algebraically closed field as fixpoints of the Frobenius map.

Lemma 6.1. Let $G$ be a finite group. Assume that $G$ has a normal series of the form

$$
1<Z(N)<N<G
$$

such that $N$ is perfect. Assume that $N$ has a cyclic complement in $G$ generated by an element t of prime power order $p^{m}$. Moreover, assume that the isomorphism type of $N$ is determined by its chief series and that $(\mathrm{ZC})_{p}$ holds for $\mathbb{Z}(G / Z(N))$. Then the isomorphism problem for $G$ has a positive solution.

Proof. Let $H$ be a group basis of $\mathbb{Z} G$. The class sum correspondence shows that there is an element $s \in H$ of the same order as $t$, the class sum of which coincides with that of $t$. Put $\bar{G}=G / Z(N)$ and let $\kappa: \mathbb{Z} G \longrightarrow \mathbb{Z} \bar{G}$ be the corresponding projection. Denote $\kappa(H)$ by $\bar{H}$. By assumption there is an automorphism $\sigma$ of $\mathbb{Z} \bar{G}$ with $\sigma(\bar{H})=\bar{G}$ and $\sigma$ fixes the class sum of $\kappa(s)$. Hence we may find an element $r \in H$ which lies in the same conjugacy class as $s$ and which has the property that $\sigma(\kappa(r))=\kappa(t)$.

Denote by $M$ the normal subgroup correspondent to $N$ in $H$. Because $\mathbb{Z} G=\mathbb{Z} H$ we know by [19] that $G$ and $H$ have the same chief series. Since $M$ and $N$ are characteristic in $H$ and $G$ respectively, the chief series of $N$ and $M$ coincide. By assumption there is an isomorphism $\omega$ between $N$ and $M$. Consider now the following commutative diagram, where $\bar{N}=\kappa(N), \quad \bar{\omega}$ is induced from $\omega$ and $\lambda$ is the composition of $\kappa$ and $\sigma$.

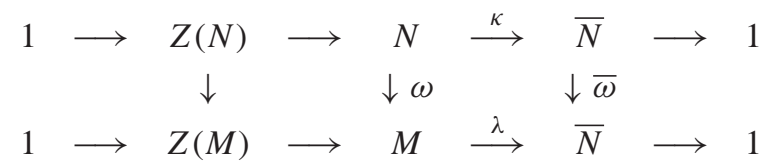

Because $N$ is perfect, each automorphism of $\bar{N}$ lifts to an automorphism of $N$. Thus we may assume that $\bar{\omega}$ is the identity.

Claim. $\omega^{-1}\left(r^{-1} \cdot \omega(n) \cdot r\right)=t^{-1} \cdot n \cdot t$ for each $n \in N$.

Proof of the claim. An easy calculation shows that

$$
\kappa\left(t^{-1} \cdot n \cdot t\right)=\kappa\left(\omega^{-1}\left(r^{-1} \cdot \omega(n) \cdot r\right)\right.
$$

for each $n \in N$. Define a map $\delta: N \longrightarrow Z(N)$ by

$$
t^{-1} \cdot n \cdot t \cdot \delta(n)=\omega^{-1}\left(r^{-1} \cdot \omega(n) \cdot r\right) .
$$


If $n \in Z(N)$ then obviously the equation holds. Moreover $\delta$ depends only on $\kappa(n)$ and thus $\delta$ induces a map from $\bar{N}$ to $Z(N)$ which is a group homomorphism. Because $\bar{N}$ is perfect we get that $\delta(n)=1$ for each $n \in N$ and the claim follows.

Define now an isomorphism $\alpha: G \longrightarrow H$ by $n \mapsto \omega(n)$ for $n \in N$ and $t \mapsto r$. The isomorphism is well defined because $t$ generates by assumption a complement to $N$.

(We thank M. Hertweck for pointing out an error in a previous version of Lemma 6.1.)

Corollary 6.2. The isomorphism problem has a positive answer for almost sporadic simple groups.

Proof. As a general reference for the facts used about the 26 sporadic simple groups we refer to [8]. If $X$ is a sporadic simple group then $|\operatorname{Out}(X)| \leqslant 2$.

Thus an almost sporadic simple group $G$ is of type $X, X .2, m . X$ or $m . X .2$. In the cases when $G$ is of type $X, X .2$ or $m . X$ it is up to isomorphism determined by its chief series. Note for this that $m=2,3,6$ or $m=12$ and that the Schur multipliers of the sporadic simple groups are always cyclic. Thus by [19] the isomorphism problem has a positive solution for $G$.

Let now $G$ be of type $m . X .2$. We use the normal series

$$
1<Z(m . X)<N=m . X<m . X .2 .
$$

Moreover, the character tables show that $X .2$ is generated by $X$ and an involution. This gives in all cases an outer automorphism of $m . X$ of order 2, and therefore a group $G$ of type $m . X .2$ such that $m . X$ has a complement of order 2. By Theorem 2.1 (ZC) holds for $X .2=G / Z(N)$. Consequently, we may apply Lemma 6.1 and show that (IP) is valid for $G$. By [28, Satz I., p. 95] there are at most two isomorphism types of groups of type m.X.2. This completes the proof.

Corollary 6.3. The isomorphism problem has a positive answer for the double covers of the symmetric groups $\mathrm{Sym}_{n}, n \geqslant 5$.

Proof. Let $\mathrm{Alt}_{n}$ be the alternating group of degree $n \geqslant 5$. By [28] the Schur multipliers of $\mathrm{Alt}_{n}$ and $\mathrm{Sym}_{n}$ are cyclic of order 2 except for $n=6,7$. The Schur multipliers of Alt 6 and $\mathrm{Alt}_{7}$ are cyclic of order 6 . In all cases $m$. Alt ${ }_{n}$ is determined up to isomorphism by its chief series. By [23] it follows that (ZC) is valid for $\operatorname{Sym}_{n}$. If $n \neq 6$ then the automorphisms of $\mathrm{Sym}_{n}$ are inner. In the case $\mathrm{Sym}_{6}$ an outer automorphism interchanges the two isoclinism classes of the double covers. Thus there is, up to isomorphism, only one double cover in this case [31, 2.21]. If $n \neq 6$ then we may apply Lemma 6.1 analogously as in Corollary 6.2. This completes the proof.

\section{References}

1. P. F. BlanchaRd, 'Exceptional group ring automorphisms for some metabelian groups, II', Comm. Alg. 25 (1997) 2735-2742. 274

2. F. M. Bleher, 'Zassenhaus-Vermutung und einfache Gruppen', Diplomarbeit, Universität Stuttgart, 1993. 281

3. F. M. Bleher, 'Tensor products and a conjecture of Zassenhaus', Arch. Math. 64 (1995) 289-298. 275, 280 
4. F. M. Bleher, 'Automorphismen von Gruppenringen und Blocktheorie', Dissertation, Universität Stuttgart, 1995. 275, 281, 284, 285, 285, 294

5. F. M. BleHer, 'Finite groups of Lie type of small rank', Pacific J. Math. 187 (1999) 215-239. 275

6. F. M. BLEHER, 'Integral group rings of finite groups of Lie type', Bull. London Math. Soc. 31 (1999) 43-44. 303

7. F. M. Bleher, G. Hiss and W. Kimmerle, 'Autoequivalences of blocks and a conjecture of Zassenhaus', J. Pure Appl. Algebra 103 (1995) 23-43. 278, 279, 280, 281

8. J. H. Conway, R. T. Curtis, S. P. Norton, R. A. Parker and R. A. Wilson, Atlas of finite groups (Oxford University Press, London/New York, 1985). 277, 304

9. M. A. Dokuchaev and S. O. JuriaAns, 'Finite subgroups in integral group rings', Canad. J. Math. 48 (1996) 1170-1179. 275

10. F. Gross, 'Automorphisms which centralize a Sylow p-subgroup', J. Algebra 77 (1982) 202-233. 302

11. M. Hertweck, 'Eine Lösung des Isomorphieproblems für ganzzahlige Gruppenringe endlicher Gruppen', Dissertation, Universität Stuttgart, 1998. 274, 275, 275, 279, 280

12. M. Hertweck and W. Kimmerle, 'On the $F^{*}$-theorem', Proceedings Groups St.Andrews in Bath 1997 I (ed. C. M. Campbell, E. F. Robertson, N. Ruskuc and G. C. Smith), London Math. Soc. Lect. Note Ser. 260 (Cambridge University Press, 1999) 346-352. 275, 302

13. B. Huppert, Endliche Gruppen I, Grundlehren der Math. Wissenschaften 134 (Springer-Verlag, Berlin-Heidelberg-New York, 1967). 278, 279

14. C. Jansen, K. Lux, R. A. Parker and R. A. Wilson, An atlas of Brauer characters, London Math. Soc. Monographs, New Series 11 (Oxford University Press, Oxford, 1995). 277, 293

15. W. KimmerLe, 'Beiträge zur ganzzahligen Darstellungstheorie endlicher Gruppen', Bayreuther Math. Schriften 36 (1991) 1-139. 276, 279

16. W. Kimmerle, 'Class Sums of p-elements', in: [26] 117-124. 275

17. W. Kimmerle, 'On Automorphisms of $\mathbb{Z} G$ and the Zassenhaus Conjectures', CMS Conference Proceedings Vol. 18 (1996) 383-397. 275

18. W. Kimmerle, 'On the characterization of finite groups by characters', The atlas of finite groups: ten years on (ed. R. Curtis and R. Wilson), London Math. Soc. Lect. Note Ser. 249 (1998) 119-138. 275, 276

19. W. Kimmerle, R. Lyons, R. Sandling and D. Teague, 'Composition factors from the group ring and Artin's theorem on orders of simple groups', Proc. London Math. Soc. (3) 60 (1990) 89-122. 275, 303, 304

20. W. Kimmerle and K. W. RogGenKAmp, 'A Sylowlike theorem for integral group rings of finite solvable groups', Arch. Math. 60 (1993) 1-6. 275

21. L. Klingler, 'Construction of a counterexample to a conjecture of Zassenhaus', Comm. Algebra 19 (1991) 2303-2330. 274

22. K. Lux, 'Algorithmic methods in modular representation theory', Habilitationsschrift, RWTH Aachen, 1997. 293 
23. G. L. Peterson, 'Automorphisms of the integral group ring of $S_{n}$ ', Proceedings AMS 59 (1976) 14-18. 276, 304

24. K. W. Roggenkamp, 'The isomorphism problem for integral group rings of finite groups', Proceedings of ICM Kyoto 1990 (Springer-Verlag, 1991) 369-380. 275 , 302, 302

25. K. W. Roggenkamp, 'Units and the isomorphism problem', Part I of [26]. 274, 279, 302

26. K. W. RoggenkAmp and M. TAYlor, Group rings and class groups, DMV-Seminar 18 (Birkhäuser, Basel-Boston-Berlin, 1992). 305, 306

27. M. SCHÖNERT et al., GAP - groups, algorithms, and programming, first edn (Lehrstuhl D für Mathematik, Rheinisch Westfälische Technische Hochschule, Aachen, Germany, 1992). 277

28. I. Schur, 'Untersuchungen über die Darstellungen der endlichen Gruppen durch gebrochene lineare Substitutionen', J.Math. 132 (1907) 85-137. 304, 304

29. L. L. Sсотт, 'Recent progress on the isomorphism problem', Proc. Symposia in Pure Math. Vol. 47 (1987) 259-274. 275, 302

30. L. L. ScotT, 'Defect groups and the isomorphism problem', Représentations linéaires des groupes finis, Proc. Colloq. Luminy, France 1988, Astèrisque 181-182 (1990) 257262. 296, 301, 302

31. M. SuzuKI, Group theory I, Grundlehren der Math. Wissenschaften 247 ( SpringerVerlag, Berlin-Heidelberg-New York, 1982). 304

32. K. WAKI, 'The projective indecomposable modules for the Higman-Sims group in characteristic 3', Comm. Algebra 21 (1993) 3475-3487. 281, 281

Frauke M. Bleher frauke@math.upenn.edu

Department of Mathematics

University of Pennsylvania

Philadelphia, PA 19104-6395

U.S.A.

Wolfgang Kimmerle kimmerle@mathematik.uni-stuttgart.de

Mathematisches Institut B

Universität Stuttgart

Pfaffenwaldring 57

D-70550 Stuttgart

Germany 